\title{
Se flageller dans la Flagellation. Enjeux et modalités de la représentation des flagellants dans leurs images de dévotion
}

Self-flagellation in the Flagellation of Christ. Stakes and modes of the representation of flagellants in their devotional images

\section{Pauline Duclos-Grenet}

\section{OpenEdition \\ Journals}

Édition électronique

URL : http://journals.openedition.org/imagesrevues/8561

DOI : 10.4000/imagesrevues.8561

ISSN : $1778-3801$

Éditeur :

Centre d'Histoire et Théorie des Arts, Groupe d'Anthropologie Historique de l'Occident Médiéval,

Laboratoire d'Anthropologie Sociale, UMR 8210 Anthropologie et Histoire des Mondes Antiques

Référence électronique

Pauline Duclos-Grenet, « Se flageller dans la Flagellation. Enjeux et modalités de la représentation des flagellants dans leurs images de dévotion », Images Re-vues [En ligne], Hors-série 9 | 2020, mis en ligne le 01 décembre 2020, consulté le 16 mars 2021. URL : http://journals.openedition.org/imagesrevues/ 8561 ; DOI : https://doi.org/10.4000/imagesrevues.8561

Ce document a été généré automatiquement le 16 mars 2021.

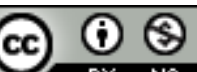

Images Re-vues est mise à disposition selon les termes de la Licence Creative Commons Attribution Pas d'Utilisation Commerciale 4.0 International. 


\title{
Se flageller dans la Flagellation. Enjeux et modalités de la représentation des flagellants dans leurs images de dévotion
}

\author{
Self-flagellation in the Flagellation of Christ. Stakes and modes of the \\ representation of flagellants in their devotional images
}

Pauline Duclos-Grenet

Durant les derniers siècles du Moyen Âge, le visage de la chrétienté fut profondément transformé par l'essor des ordres mendiants et par l'investissement croissant des laïcs dans la sphère religieuse. En effet, à partir du $\mathrm{XI}^{\mathrm{e}}$ et plus encore $\mathrm{du} \mathrm{xII}^{\mathrm{e}}$ siècle, le laïcat revendiqua une autonomie croissante en matière de spiritualité qui trouva, dans le mouvement confraternel, un espace d'expression et d'action à la fois dévotionnelles et caritatives ${ }^{1}$. Les nombreuses confréries qui essaimèrent partout en Occident et en particulier en Italie, furent l'une des manifestations les plus importantes des aspirations nouvelles du laïcat. Dès les origines, ces

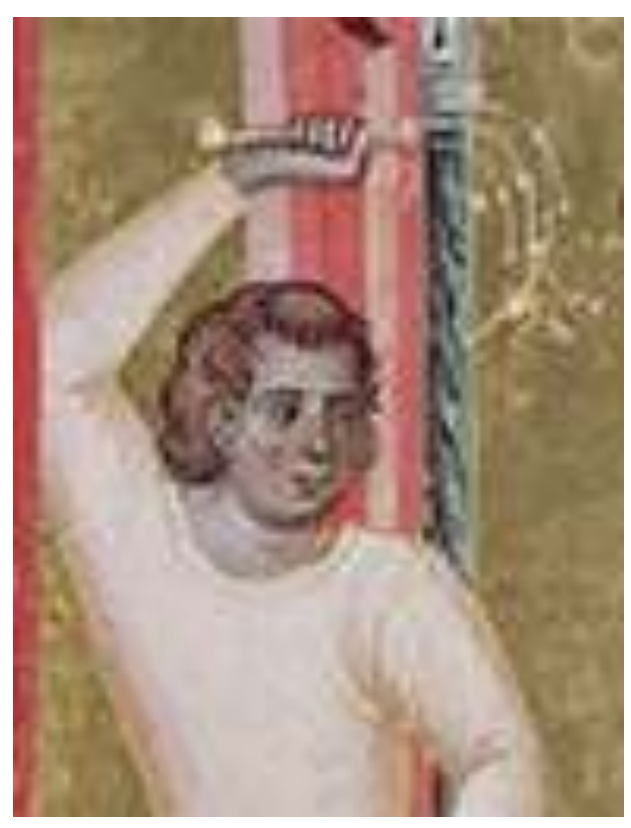
institutions commencèrent à se spécialiser pour devenir des confréries mariales, pénitentes, ou charitables ${ }^{2}$. Les pratiques des confréries pénitentes accueillirent, au milieu du XIII ${ }^{\mathrm{e}}$ siècle, une pratique, nouvelle pour les laïcs : la discipline, ou flagellation. Acte pénitentiel à l'origine confiné 
aux milieux érémitiques puis monastiques, devint également une ascèse corporelle encourageant la mémoire et l'imitation de la Passion du Christ ${ }^{3}$. L'imitation du modèle christique comme voie vers le salut trouva en saint François, présenté par l'ordre franciscain comme un Alter Christus, un second Christ, ayant reçu dans sa chair les stigmates de la Passion, son expression la plus parfaite. Si ces confréries assumèrent une distance vis-à-vis de l'autorité ecclésiastique, elles entretinrent un lien étroit avec les nouveaux ordres mendiants dont elles partageaient les aspirations spirituelles, des formes de dévotions, des pratiques et, au moins dans un premier temps, des lieux de culte ${ }^{4}$. En outre,

«l'identification au Christ souffrant dans un corps perclus de douleurs et ensanglanté permit alors aux fidèles de se sentir eux-mêmes investis d'une mission rédemptrice, sans l'intermédiaire d'un mandat ecclésiastique $»^{5}$.

Aussi, les Flagellants et leurs confréries furent l'objet de la méfiance et parfois de la condamnation des autorités ecclésiastiques et civiles, et la pratique disciplinaire, fermement encadrée et limitée. En 1349, le pape Clément vi condamna ainsi les excès des mouvements flagellants dans la bulle Inter sollicitudines ${ }^{6}$.

3 Comme leurs homologues, les confréries de disciplinés ont eu, sous l'influence de la spiritualité mendiante, largement recours aux images. Outre les traditionnelles missions d'enseignement des vérités divines et de remémoration des exemples chrétiens, les images se voient chargées, par Thomas d'Aquin notamment, de la fonction de susciter l'émotion du fidèle et partant, de stimuler une dévotion plus affective et empathique ${ }^{7}$. L'image, indissociable de sa matérialité, c'est-à-dire de son corps, entretient avec le fidèle un rapport spéculaire dans lequel expériences visuelle et corporelle sont intimement liées ${ }^{8}$.

Dans ce contexte, les images de la Flagellation du Christ occupent, chez les confréries, une place particulière, puisque cette dernière incarne autant qu'elle justifie leur identité. En outre, dans les statuts, sur les bannières ou les retables, les flagellants se font fréquemment représenter en train de s'infliger la discipline, au pied d'un Christ flagellé. Il s'agira ici, après avoir étudié la place des images dans les statuts, de cerner le rôle particulier de la figuration de la Flagellation dans l'ensemble des images des confréries flagellantes. Enfin, nous nous demanderons comment ces images singulières de flagellants dans la Flagellation, peuvent être considérées comme une mise en image du processus de memoria par l'imitation.

\section{Images et dévotion dans les chroniques et les statuts des confréries}

5 Le phénomène flagellant est né très précisément en $1260^{\circ}$, quand, sous l'impulsion de l'ermite franciscain Raniero Fasani, est lancée depuis Pérouse, la première grande procession de disciplinés. Hommes, femmes, enfants de tous âges et toutes classes sociales confondues, entrent dans les villes aux cris de "Misericorde et Paix" ("Misericordia e Paze »), pour demander le retour de la paix civile. Cette manifestation se reproduit et s'étend à de nombreuses cités, jusqu'à Rome, en passant par la Toscane, et vers le Nord (Lombardie et Vénétie). C'est à la suite de ces manifestations que naissent des confréries flagellantes, dont les membres sont diversement qualifiés de flagellants, disciplinés, battus, ou encore recommandés (flagellanti, disciplinati, battuti ou 
raccomandati). Ces institutions connaissent un vif succès et ne cessent de se multiplier jusqu'au $\mathrm{xv}^{\mathrm{e}}$ siècle $^{10}$.

Les nombreuses sources qui éclairent tant le phénomène flagellant que la vie des confréries témoignent d'un recours massif aux images dans leurs pratiques dévotionnelles, publiques ou privées. Ainsi, les chroniques qui relatent les processions publiques consignent le spectacle étonnant de ces fidèles qui, dévêtus jusqu'à la taille, se frappent le dos et le torse en poussant des cris ou en chantant prières de louanges (laudi) et psaumes pénitentiels. Diverses images scandent la procession. Les Annales d'un anonyme padouan ${ }^{11}$ relatent, par exemple, que

«de jour, comme de nuit, munis de cierges allumés, par centaines et milliers et même dizaine de milliers, (les flagellants) allaient de ville en église, et se prosternaient humblement devant les autels, précédés par des prêtres portant des croix et des bannières $»^{12}$.

7 Salimbene de Adam dans sa Cronica, dresse le même tableau ${ }^{13}:$ les autorités cléricales et municipales accompagnent les processions, encadrées par des étendards. Ces chroniques sont parfois agrémentées d'une illustration fidèle aux descriptions. Au folio 618 de la Chronique du lucquois Giovanni Sercambi (1348-1424), est représentée la rencontre de deux processions au sortir d'églises ${ }^{14}$. En tête de ces deux branches humaines, deux crucifix sculptés, cernés de candélabres, sont présentés par les membres qui ouvrent la marche. Les frères sont agenouillés, vêtus d'une robe blanche sur laquelle se détache une croix, au niveau de l'épaule, insigne de la compagnie. Les visages inclinés sont en partie dissimulés par un capuchon rabattu sur le front. Des mains croisées sur la poitrine, pendent des flagella (fouets ${ }^{15}$ ), constitués de deux cordes nouées à plusieurs reprises ou lestées de boules de plomb. Dans le manuscrit contenant la Chronique de Giovanni Villani, le folio 197 met également en scène une procession, de manière plus sommaire ${ }^{16}$. Six figures se succèdent, dénudées jusqu'à la taille. De la main droite, elles se frappent l'épaule gauche et le dos, au moyen de ce qui semble être un bouquet de brindilles. En tête de la suite, et surtout en haut de l'image, se dresse une croix orfévrée agrémentée d'un étendard dont le motif est précisément représenté : un Christ, mains liées derrière le dos, est frappé par deux bourreaux. Outre ces étendards et ces croix processionnelles ou crucifix, il est également fait mention dans les textes de petits panneaux peints (tavole $)^{17}$.

8 Les statuts des confréries renseignent eux aussi sur la présence des images dans les rites confraternels ${ }^{18}$. Les formules les plus fréquentes concernent la mention d'une image pieuse et de la révérence qui lui est due. Ainsi, à trois reprises, les textes des Disciplinati de Borgo Porta Nova de Vicence, mentionnent l'existence d'une image peinte, devant laquelle chacun est systématiquement tenu de s'incliner :

«Les hommes de ladite congrégation doivent révérer des images des saints quand ils seront arrivés devant elles. De même, nous statuons et ordonnons que toujours, quand un membre de la congrégation se trouve devant une image de Notre Seigneur Jésus Christ et de la bienheureuse Vierge Marie sa mère, il doive s'incliner en révérence devant elles $»^{19}$.

La deuxième occurrence s'inscrit dans un contexte différent. Alors qu'il est question d'encourager les membres à adopter une attitude décente quand ils circulent en ville (" quando vadunt per civitatem »), le texte les engage à s'incliner devant l'image de saints («coram picturam sanctorum ») ${ }^{20}$. Enfin, le paragraphe 24 est quant à lui exclusivement consacré à la question de l'attitude à adopter face aux images : «De inclinatione facienda coram Crucifixo et virgine Maria » (« De l'inclination qu'il faut effectuer devant le crucifix 
et la Vierge Marie »). Une fois encore, il est du devoir des membres d'incliner le chef avec révérence «cum reverentia caput inclinare »" Dans ces extraits, si l'on excepte les images des saints qui se trouvent à l'extérieur de la confrérie, il est donc fait mention de trois types d'images : celles du Christ, de la Vierge, et d'un crucifix ${ }^{22}$.

On trouve, dans les statuts de la confrérie flagellante de San Niccolò à Palerme, des indications encore plus précises:

« (La confrérie) doit en outre avoir une enseigne belle et dévote, figurant le Christ et la porter en procession, ainsi qu'un sceau présentant en son centre le Christ flagellé, et autour, la devise de la compagnie $\aleph^{23}$.

11 Ainsi, la confrérie doit se doter d'une enseigne de procession représentant le Christ et d'un sceau qui doit rappeler l'activité de la confrérie. Plus encore, l'organisation de l'image est prescrite : la devise de la compagnie doit entourer le Christ flagellé. Un deuxième exemple fait également mention d'une image de la Flagellation. Les règles de la Compagnia di S. Maria dei Battuti de Modène ${ }^{24}$ font cette fois référence à un étendard représentant une Flagellation.

"Sur (son) étendard, on peut contempler l'image de notre Sauveur Jésus Christ flagellé à la colonne, en mémoire de sa passion très cruelle, car cette confrérie a pour nom la Compagnie du Christ flagellé $»^{25}$.

12 Nous l'avons vu, dans la Chronique de Giovanni Villani, les pénitents progressent derrière un étendard représentant une Flagellation. Les extraits précédents témoignent que le choix iconographique est un enjeu suffisamment important pour qu'il figure dans les statuts. Il reste à en expliciter la nature. Dans le cas du sceau ou de l'étendard, il s'agit dans un premier temps de créer une image à caractère public de la confrérie, une image officielle, qui la représente, l'engage moralement, et la rende visible et reconnaissable par d'autres. Enfin, il arrive, mais encore plus rarement, que les statuts délivrent des informations sur l'usage des images durant l'office et l'administration de la discipline: les statuts de San Michele Arcangelo de Florence, rédigés en 1415, stipulent que la confrérie doit se réunir dans un lieu où se trouve une image du Christ (" et dans ce lieu, ils conservent une image du Christ») ${ }^{26}$. Quand les membres sont rassemblés, l'un d'entre eux s'avance, devant l'image du Christ (« dinançi alla imagine di cristo »), lève ses mains jointes et entame le Adiutorium nostrum ${ }^{27}$. C'est devant l'image $\mathrm{du}$ Christ, en lui adressant des gestes et des paroles que le rituel est amorcé. Celle-ci est donc primordiale, dans le temps du rituel comme dans sa condition. On ramasse les disciplines posées au sol, tandis que l'officiant récite un verset du psaume 2, "Apprehendite disciplinam», ( Emparez-vous de la discipline»). À ces mots, les pénitents s'administrent les coups. Cette réponse en acte au verset repose sur une traduction littérale de l'injonction psalmique, comprise comme performative ${ }^{28}$. Apprehendite disciplinam est en effet la traduction proposée dans la Vulgate, à partir des textes grecs et arméniens. Ce n'est qu'au XvII siècle que les études philologiques postérieures ont rétabli la traduction à partir du texte hébreu et le verset se traduit désormais par « Embrassez le Fils». 
Figure 1

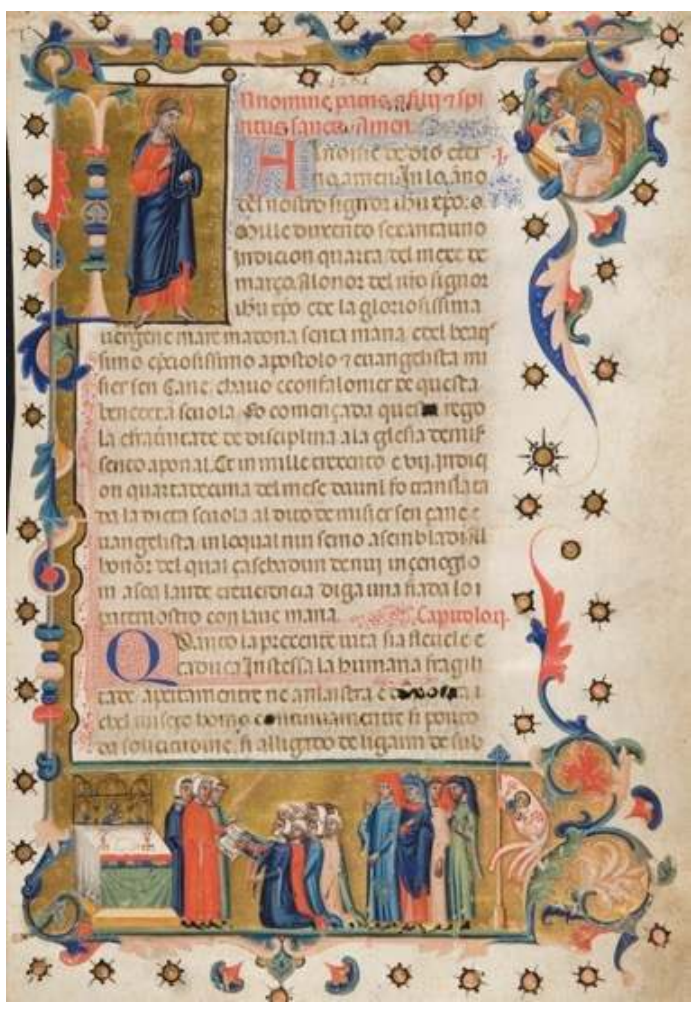

Mariegola de la Scuola di San Giovanni Evangelista de Venise, milieu du xive siècle, tempera sur parchemin, Venise, Fondation Cini, inv. 2041. 
Figure 2

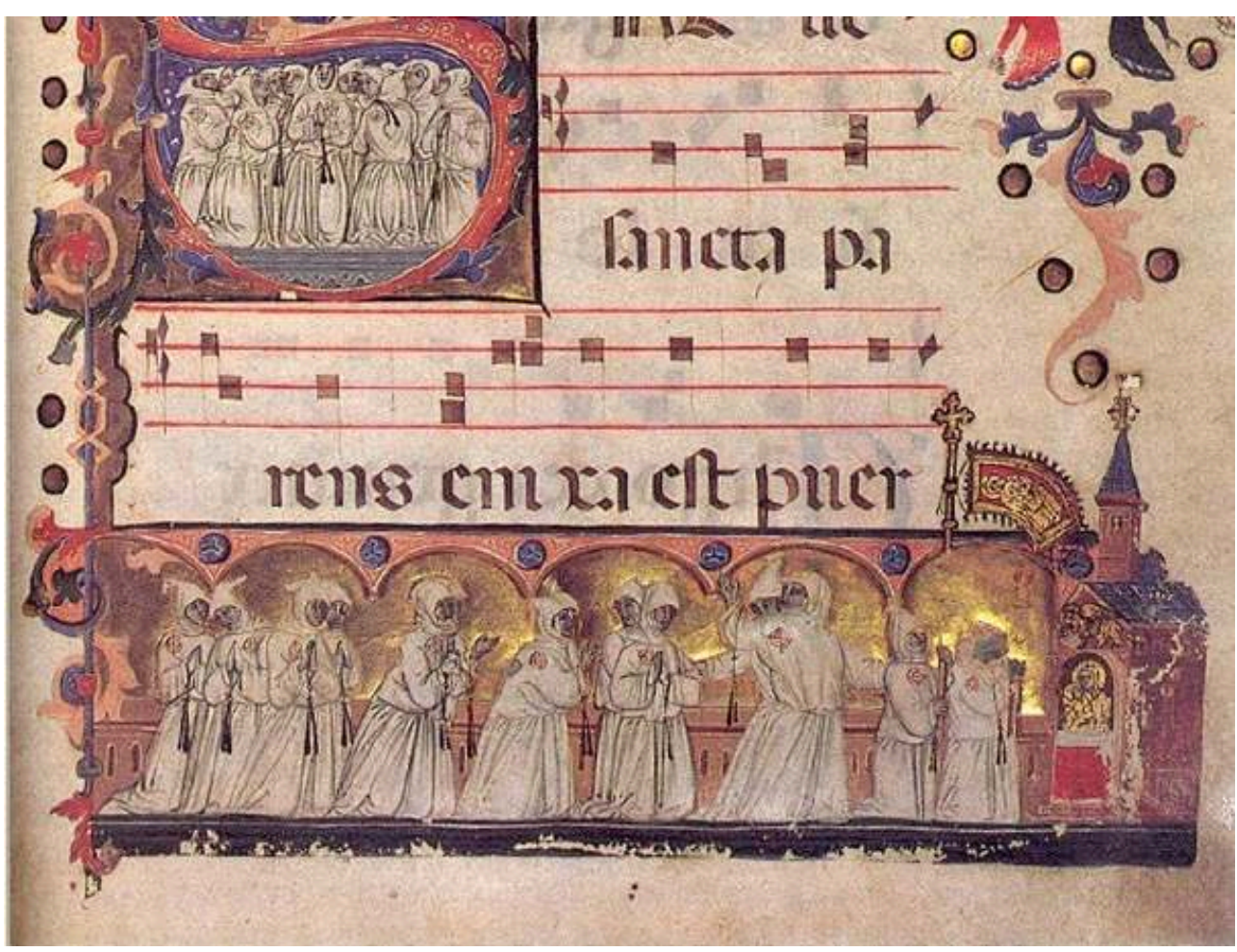

Graduel de la Scuola di Santa Maria della Carità, milieu du XIVe siècle, tempera sur parchemin, Venise, BNM, cod. lat. II, 119, fol. 1R.

Tiré de L. Humphrey, The Illumination of the Confraternity, op.cit., pl. 19d.

Figure 3

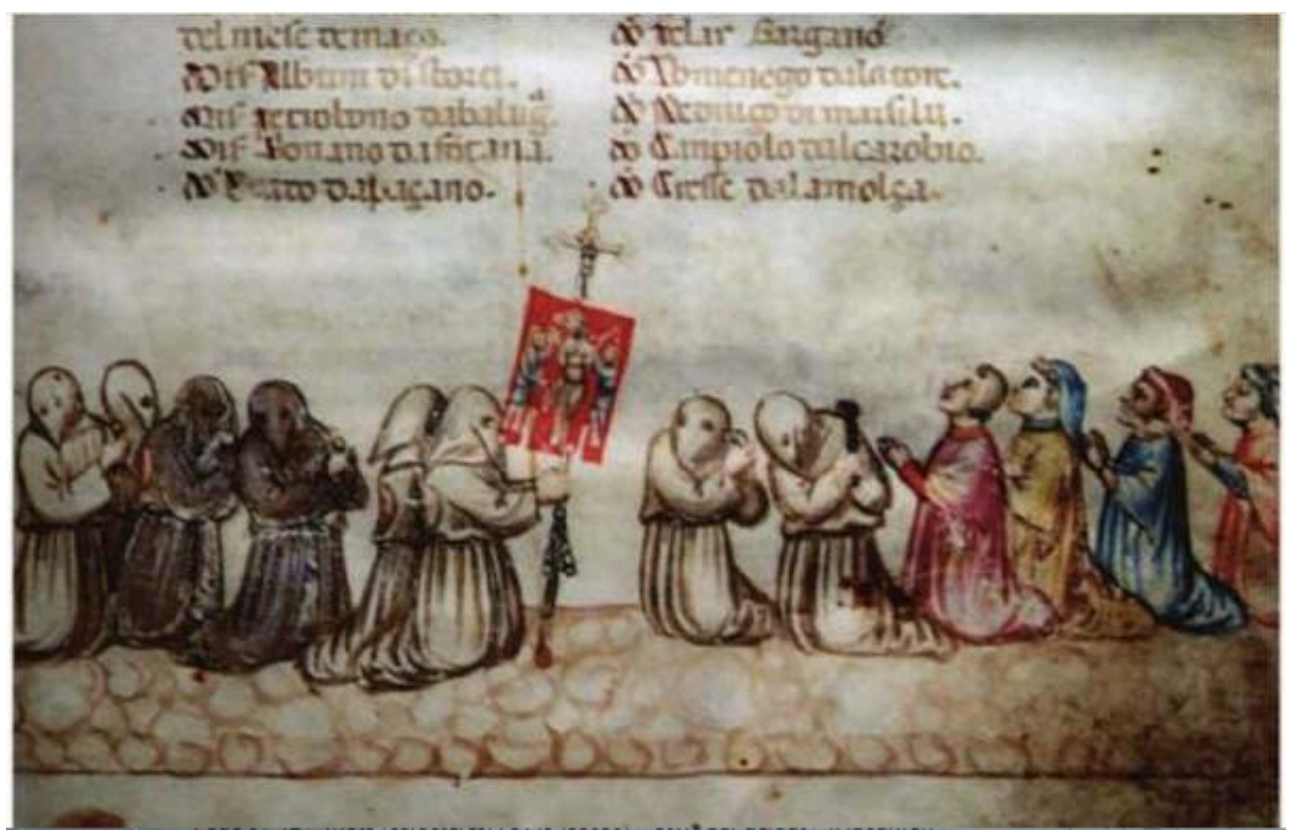

Statuts de la Confraternita della Carità de Modène, dernier quart du xı e siècle, tempera sur parchemin, Modène, Bibliothèque Estense, ms. 2, dernier quart du xiv ${ }^{e}$ siècle. 
Les indications, somme toute laconiques des sources statutaires, sont corroborées et précisées par l'iconographie. Fréquemment illustrés, les statuts présentent à l'aide de l'image l'accompagnement visuel de la confrérie, constitué de retables, de tavole, de livres ou d'étendards. Consistant en une mise en abyme dans un contexte liturgique ou para-liturgique fictif, ces "méta-images", ainsi que leur fréquence, témoignent de l'importance des images dans la vie dévotionnelle des battuti. La marge inférieure du préambule de la règle de la Scuola di San Giovanni Evangelista de Venise (1340-1355) présente un dispositif d'images étoffée ${ }^{29}$ (fig. 1). S'y déroule la cérémonie d'accueil des nouveaux membres, agenouillés au centre, tandis qu'on leur présente le livre des statuts. Les novices prêtent serment devant l'autel surmonté d'un retable compartimenté représentant, sur deux registres, une Vierge à l'Enfant et un Christ de douleurs (Vir dolorum), cernés de saints. Deux calices sont disposés sur l'autel et l'étendard situé à droite de l'image, derrière le groupe, arbore saint Jean Évangéliste. La scène et les figures sont donc littéralement encadrées par les images : à gauche, le retable sur l'autel, au centre, les statuts illustrés, à droite, l'étendard ${ }^{30}$. Les folios consacrés au chant dans les statuts sont également illustrés. Leurs images sont d'autant plus intéressantes qu'elles ne représentent plus la cérémonie d'accueil des novices, mais le moment de la célébration, et peut-être de la discipline. Ainsi en est-il pour le folio 1r du Graduel de la Scuola di Santa Maria della Carità ${ }^{31}$ (fig. 2). En bas de la page, suivant le déploiement linéaire des neumes, se succèdent des pénitents en procession. Ils sont agenouillés, mains jointes, devant l'image posée sur l'autel d'une église, une Vierge à l'Enfant. Les membres à la tête du cortège tiennent, pour l'un, une croix processionnelle dotée d'un étendard représentant lui aussi une Vierge à l'Enfant; pour les deux autres, les objets sont devenus illisibles. Il s'agissait peut-être d'un crucifix ou de candélabres. Les hommes, vêtus de blanc, le capuchon laissant apparaître le visage, ne sont pas précisément en train de s'infliger la discipline. Mais les flagella, pendant au poignet de chacun, explicitent la pratique de la discipline pour cette confrérie de charité. Le dernier exemple représente lui aussi la dévotion au moment de la procession. Dans la marge inférieure de la table des matières des statuts de la Confraternita della Carità de Modène ${ }^{32}$ (fig. 3), se succèdent de simples fidèles et des flagellants, vêtus tantôt de noir, tantôt de blanc, les premiers en position de prière, les autres se livrant à la discipline. Au centre, deux frères tiennent une croix processionnelle et un étendard rouge, sur lequel est, de manière très visible, représenté un Christ à la colonne torturé par deux bourreaux. Les deux membres ne peuvent s'infliger les mortifications de rigueur, mais le premier tient une double chaîne qui pend ainsi, dans le prolongement de l'image-objet composé par le corps du Christ sur l'étendard, et terminé par la croix. Quant au second, l'encoche sur son vêtement laisse apparaître une chair mutilée et sanglante. Cette image oppose ainsi clairement deux formes de dévotion. Les figures vêtues « en civil » se livrent à la méditation les mains jointes, la tête renversée, et le regard dirigé vers le texte qui les surplombe. En face d'eux, les professionnels de l'imitatio s'exécutent au pied d'une image du Christ luimême flagellé. Cette miniature met en image la spécialité de la confrérie et sa pratique de la discipline. Mais l'image va plus loin. Elle affirme ce que les textes ne font que timidement : le rôle nécessaire de l'image comme support de la dévotion, fondée sur l'imitatio Christi. 


\section{La polyvalence de la Flagellation du Christ au sein du dispositif imagier flagellant}

14 Si les textes et illustrations des statuts des confréries témoignent de l'importance de l'image pour l'identité et la vie spirituelle de ces institutions, ils ne parviennent pas à en restituer la grande variété. En revanche, l'étude des inventaires et des sources matérielles permet de cerner un large spectre d'images, dont les usages variés et multiples esquissent les contours d'un dispositif complexe d'images ${ }^{33}$. Nous appellerons apparatus ce dispositif d'images, mis en œuvre ou activé par les membres des confréries, pour des usages divers et non exclusifs. Cette mise en relation d'images fait par ailleurs émerger un réseau iconographique au sein duquel la Flagellation de Jésus tient une place à la fois importante et singulière.

\section{Figure 4a}

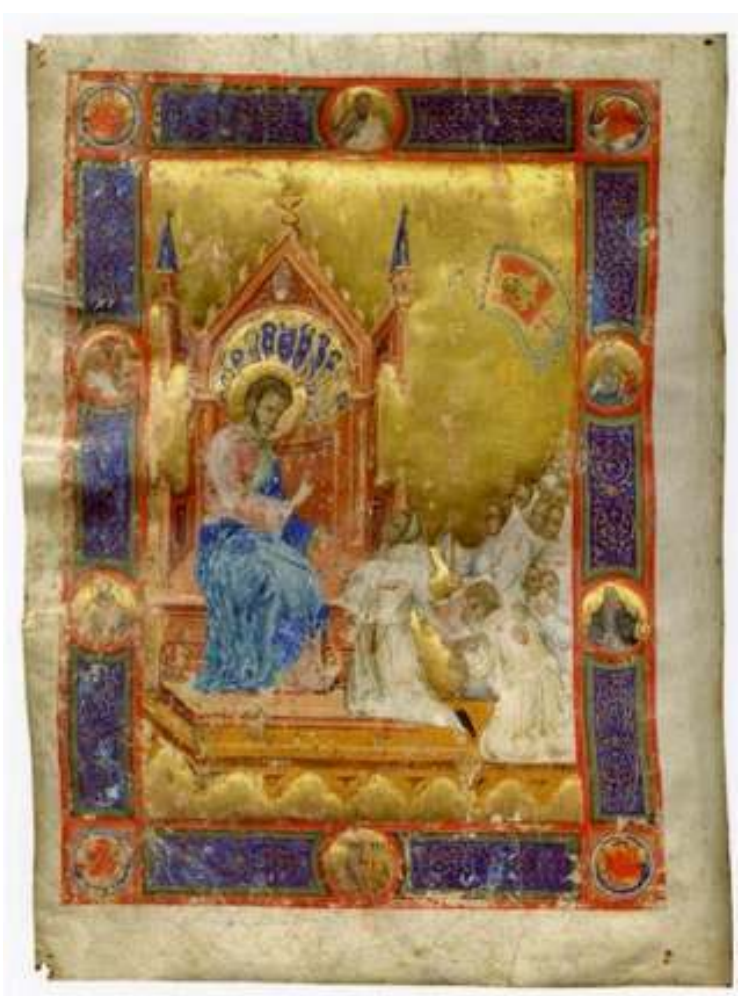

Statuts de la Scuola di San Marco, Saint Marc remettant la règles aux membres et Flagellation du Christ, milieu du xive siècle, tempera sur parchemin, Venise, BMC, ms. IV. Fol. 83.

Tiré de L. Humphrey, The Illumination of the Confraternity, op. cit., pl. 12. 
Figure 4b

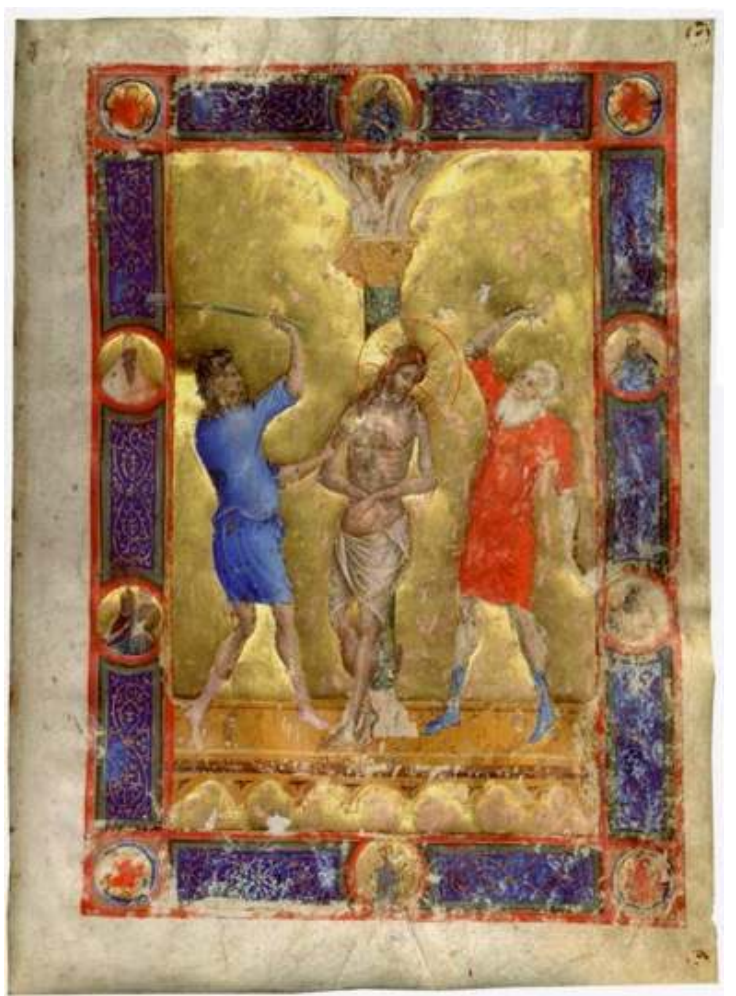

Statuts de la Scuola di San Marco, Saint Marc remettant la règles aux membres et Flagellation du Christ, milieu du xive siècle, tempera sur parchemin, Venise, BMC, ms. IV. Fol. 83.

Tiré de L. Humphrey, The Illumination of the Confraternity, op.cit., pl. 12.

Certaines images assument un caractère officiel et participent à l'élaboration de l'identité de la confrérie, au sein de l'espace social. Pour le cas des confréries flagellantes, les images de la Flagellation endossent de manière particulièrement adéquate cette fonction. Comme les portes des oratoires des confréries de charité surmontés d'un relief représentant une Vierge de Miséricorde, ou celles des laudi avec une Vierge à l'Enfant ou une Annonciation, les confréries flagellantes peuvent orner le couronnement de leur lieu de réunion d'images sur lesquelles des pénitents se flagellent. Dans les sceaux en revanche, la Flagellation est directement représentée, comme marque identitaire et symbolique de la confrérie. Nous l'avons vu, les statuts peuvent préciser ce choix iconographique. Dans son analyse des sceaux (sigili) utilisés par les confréries flagellantes, Giacomo Bascapé montre que, parmi des solutions iconographiques diverses, les confréries se tournent volontiers vers des images du Christ flagellé ou crucifié ${ }^{34}$. Ces sigilli, précise l'auteur, étaient également des insignes arborés sur la poitrine par les membres de la compagnie, donnant à l'objet un double caractère, officiel et publicitaire : «de telles figurations, par leur évidence immédiate, répondaient à la nécessité d'être rapidement reconnaissables, pendant que l'efficacité de la suggestion devait exciter chez les chrétiens la piété et la prière $»^{35}$. Aussi, le choix iconographique du Christ flagellé est-il fréquent, et Giacomo Bascapé en mentionne un certain nombre dans son étude. Le sigillo de la confrérie des pénitents de Crémone présente une composition complexe. Une devise ("sigillum societatis sancte discipline iesu christi de cremona », le sceau de la compagnie de la sainte discipline de Jésus-Christ de Crémone ${ }^{36}$ ) se déploie sur le pourtour d'une image divisée en deux : un Christ flagellé 
par deux bourreaux surplombe un flagellant agenouillé devant une sainte. Sont ainsi mis en regard à la fois l'objet de la dévotion de la confrérie, ainsi que sa pratique dévotionnelle, fondée sur l'imitation des souffrances du Christ. Il en va de même pour l'illustration des statuts de confrérie. Ceux de la Scuola di San Marco à Venise, datant du milieu du XIV siècle, présentent en frontispice la double identité de la communauté sur une double page: l'évangéliste Marc remettant aux membres la règle et en face, la Flagellation du Christ entre deux bourreaux (fig. $4 \mathrm{a}$ et $4 \mathrm{~b})^{37}$.

Figure 5

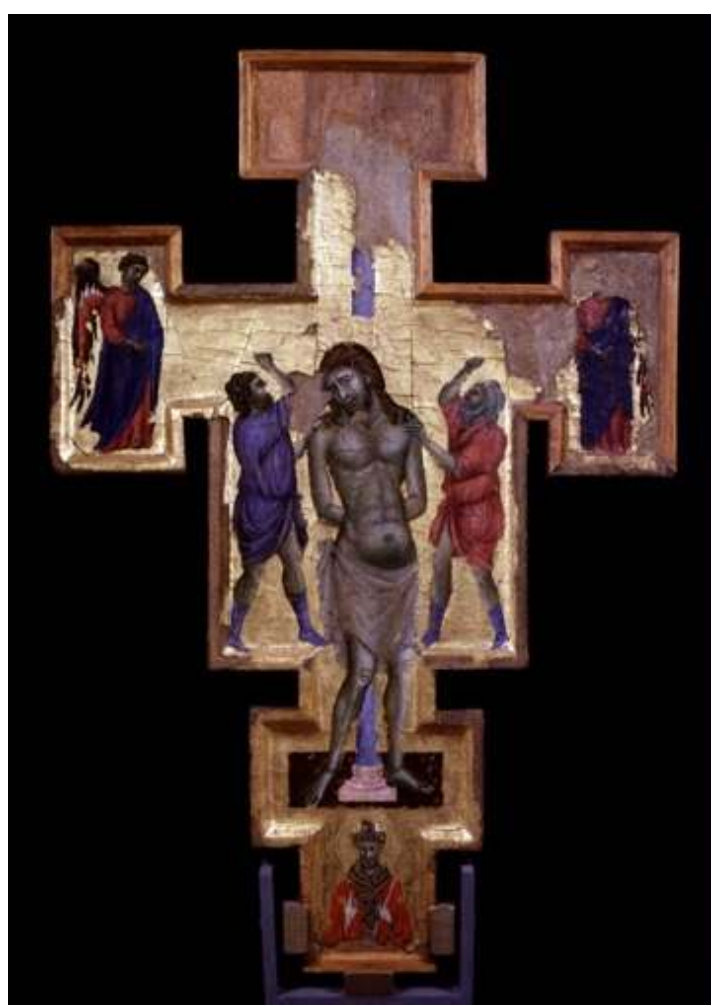

Maestro della Croce di Gubbio, croix peinte, Flagellation et Crucifixion, vers 1285-1290, tempera sur bois, $55 \times 38$ cm, Pérouse, Galleria Nazionale dell'Umbria.

Étendards et croix processionnelles, images mobiles, participent de la définition visuelle des confréries et mettent également à l'honneur la Flagellation. La Galleria nazionale dell'Umbria de Pérouse conserve l'une de ces croix processionnelles, considérée comme un unicum ${ }^{38}$. Il s'agit d'une croix exécutée vers 1285-1290 par celui que la critique a nommé le Maestro della croce di Gubbio pour la Confraternita dei Disciplinati de Pérouse, la plus ancienne de toutes, fondée dès 1260. L'originalité de cette croix repose sur son iconographie (fig. 5). En effet, dans les exemples qui nous sont parvenus, soit les croix sont non-figuratives, soit elles représentent un Christ en croix. Ici, si l'une des faces est bien une Crucifixion, le revers est en revanche occupé par une Flagellation ${ }^{39}$. Au revers, Jésus est donc lié à la colonne, entouré de deux bourreaux, l'un d'âge mûr, vêtu de bleu, l'autre plus âgé, vêtu de rouge. Ils tiennent Jésus dont les mains sont placées dans le dos, directement liées à la colonne. Les deux faces de l'image mettent en œuvre un jeu fait d'échos et de contrastes. À la branche verticale de la croix répond la colonne. La palette chromatique plus étoffée de la Crucifixion se réduit au bleu et au rouge dans la Flagellation. Au corps brisé du Christ mort répond le corps 
vigoureux, légèrement déhanché d'un Jésus bien vivant qui interpelle l'observateur de son regard intense. Cette image se comprend dans son contexte fonctionnel: la Crucifixion ouvre la marche, comme il est de rigueur, et constitue un appel public à la componction et à la contrition. Mais la Flagellation, que voient donc les participants actifs de la procession, est une invitation à se remémorer et à rejouer les souffrances de la Passion. Cette face est un support visuel pour l'action des flagellants qui la suivent.

\section{Figure 6a}

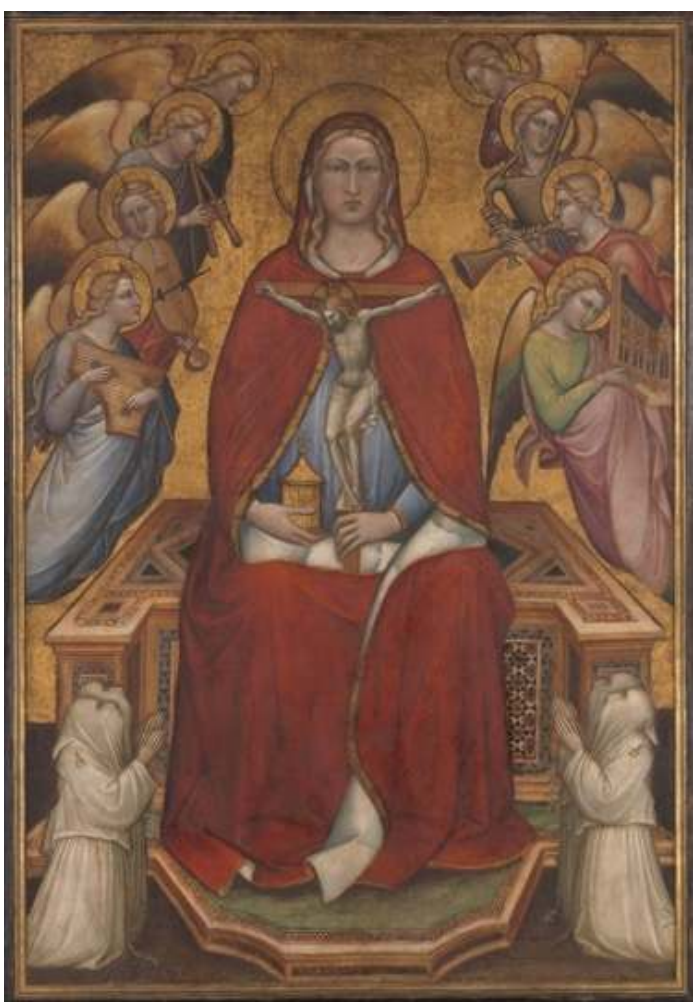

Spinello Aretino, étendard processionnel, Madeleine trônant et Flagellation, vers 1395, tempera sur bois, 176,5×120 cm, New York, Metropolitan Museum of Art. 


\section{Figure 6b}

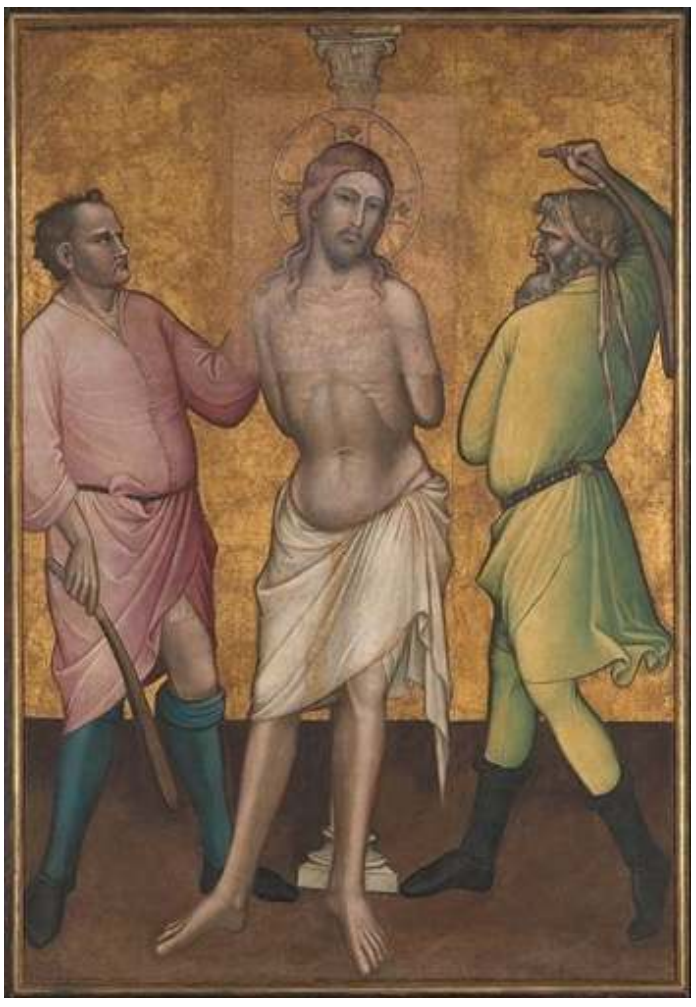

Spinello Aretino, étendard processionnel, Madeleine trônant et Flagellation, vers 1395, tempera sur bois 176,5×120 cm, New York, Metropolitan Museum of Art. 


\section{Figure $7 a$}

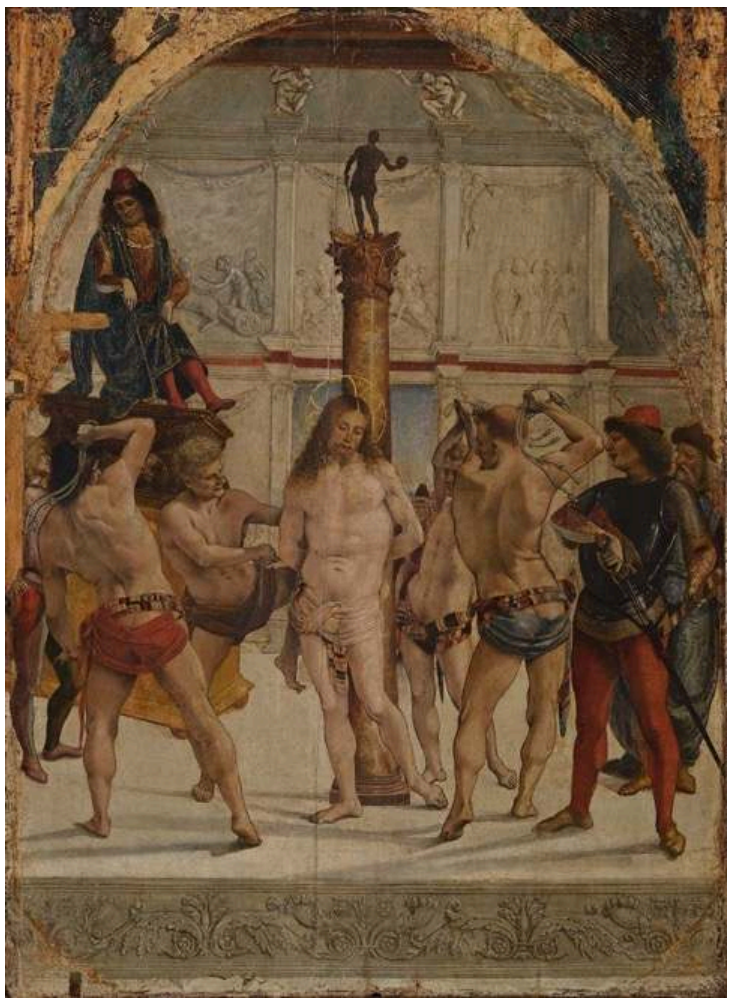

Luca Signorelli, étendard processionnel, Flagellation et Vierge allaitant l'Enfant, vers 1482-85, tempera sur bois, 84×60 cm, Milan, Pinacothèque Brera, inv. 327-347. 
Figure $7 \mathrm{~b}$

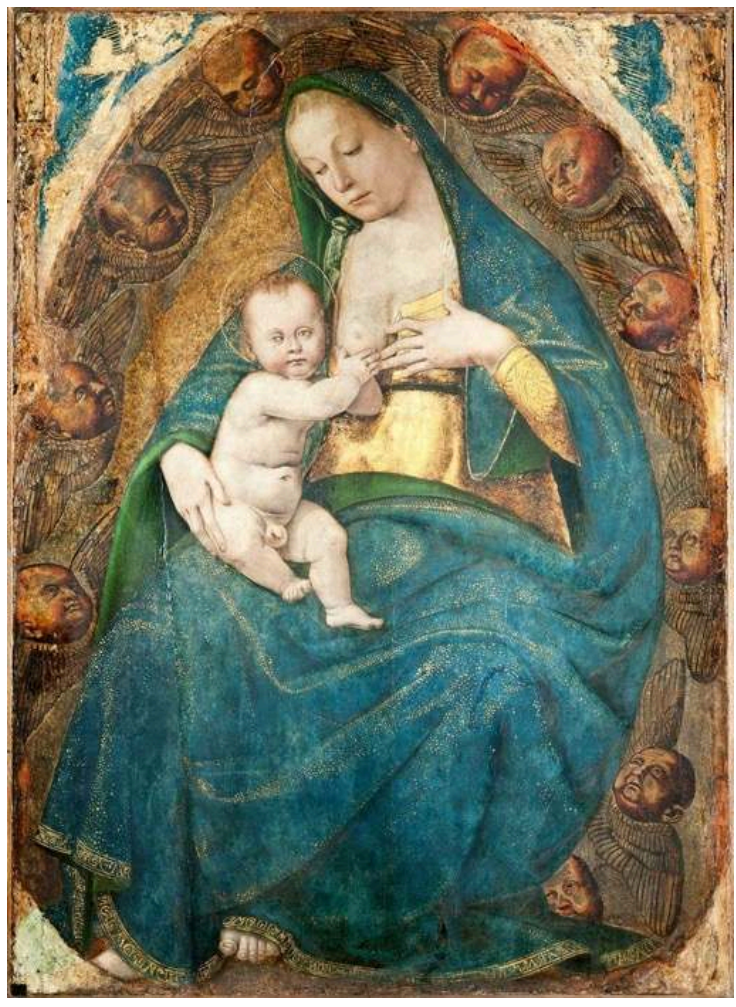

Luca Signorelli, étendard processionnel, Flagellation et Vierge allaitant l'Enfant, vers 1482-85, tempera sur bois, 84×60 cm, Milan, Pinacothèque Brera, inv. 327-347. 
Figure 8a

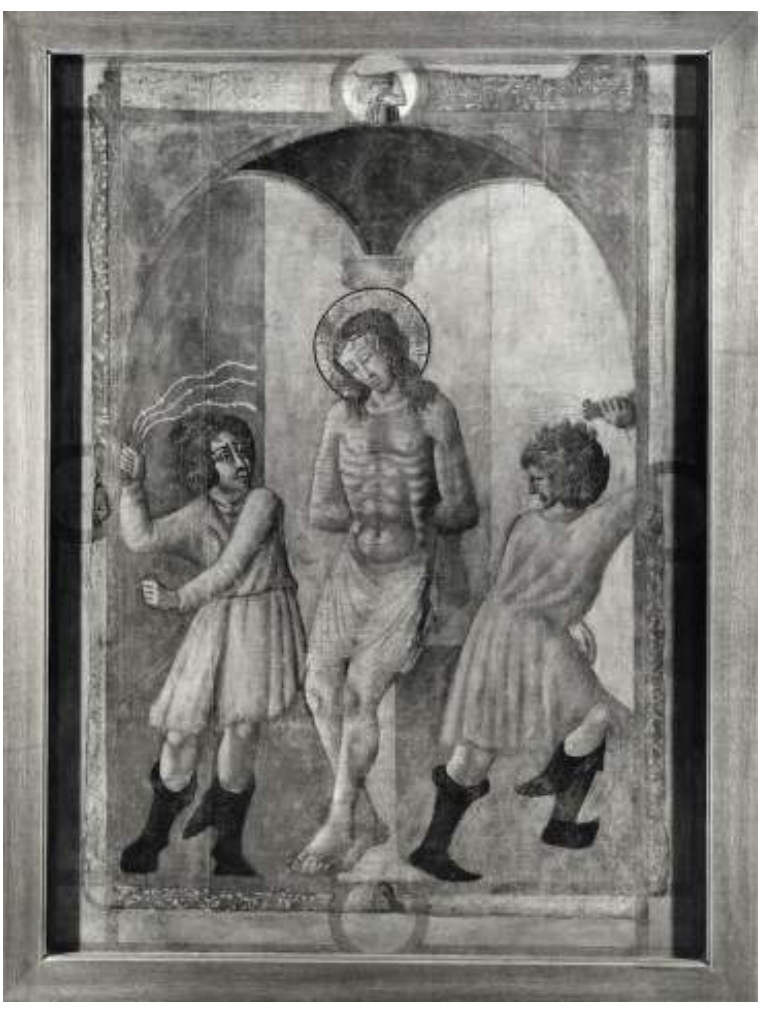

Carlo di Giovanni, étendard processionnel, Flagellation et Crucifixion, 1445-1458, tempera sur toile, Montisi, oratoire Sant'Antonio abate della compagnia del SS. Sacramento.

Tiré de la Fototeca de la Fondazione Zeri, nº 15066. 
Figure 8b

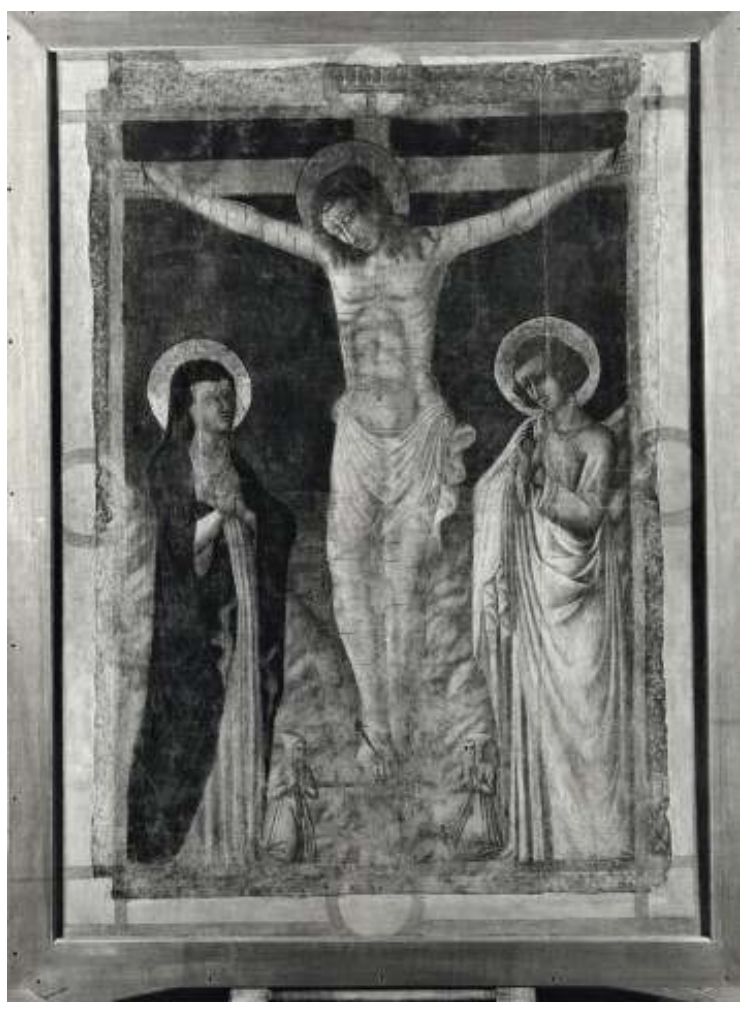

Carlo di Giovanni, étendard processionnel, Flagellation et Crucifixion, 1445-1458, tempera sur toile, Montisi, oratoire Sant'Antonio abate della compagnia del SS. Sacramento.

Tiré de la Fototeca de la Fondazione Zeri, nº 15066.

La plupart des étendards, qu'ils soient en tissu ou en bois, quand ils ne se limitent pas à représenter l'emblème de la confrérie, jouent le même rôle injonctif. Généralement, les panneaux de bois sont peints sur les deux faces et adoptent pour programme, la formule que nous avons trouvée plus haut sur la croix processionnelle : une Crucifixion et une Flagellation. Spinello Aretino introduit une variante pour la bannière de Santa Maria Maddalena de Borgo San Sepolcro. À la Crucifixion est substituée la sainte patronne présentant un crucifix et formant un trône de grâce (fig. $6 \mathrm{a}$ et $6 \mathrm{~b})^{40}$. De même, l'étendard de Luca Signorelli, exécuté des années 1475 pour la confrérie dei Raccomandati de Fabriano allie une Flagellation et une Vierge allaitant l'Enfant (fig. 7a et $7 b$ ). L'étendard de toile exécuté par Carlo di Giovanni au milieu du $\mathrm{Xv}^{\mathrm{e}}$ siècle pour la Compagnia del Santissimo Sacramento à Sienne, constitue en revanche un exemple de l'iconographie la plus courante associant la Crucifixion à la Flagellation (fig. 8a et 8b) ${ }^{41}$. Comme pour les frontispices des statuts, l'image a la sobriété et le dépouillement d'une imago pietatis. Comme si Jésus était déjà mort, ses yeux sont clos, la tête inclinée, dans une posture de l'Homme de douleurs ${ }^{42}$. La représentation est " mise en cadre », comme une image dans l'image : un premier cadre, fait d'une bande bicolore couronnée par un médaillon dans lequel on distingue le buste d'un frère mineur, crée une première structure. Dans l'image figurative proprement dite, un premier seuil est représenté, au moyen de la figuration d'un arc en berceau, qui donne à l'action un statut in medias res. Enfin, les figures de la Flagellation semblent se tenir dans l'espace ménagé par la profondeur du champ. La colonne est l'élément d'articulation des deux espaces, à la fois sur la bordure de l'image et dans sa profondeur. Nous y reviendrons. 
Parmi les objets liturgiques, certains inventaires, notamment ceux de la Compagnia di Gesù Pellegrino à Florence en 1350 étudiés par Kathleen G. Arthur, mentionnent l'existence d'une petite colonne destinée à être placée sur l'autel, à côté du crucifix ${ }^{43}$. La colonne est une nouvelle fois mentionnée dans les inventaires de 1421, incidemment, à propos d'un objet situé "devant l'autel, à côté de la colonne »" L'auteur en conclut ainsi qu'au début du $\mathrm{xv}^{\mathrm{e}}$ siècle, dans un contexte de diffusion de la dévotion envers la croix et la colonne, la confrérie disposait d'un autel sur lequel était placée une colonne. Par le passé, ces deux objets ont été compris comme des images abstraites. Or, elles sont au contraire littérales et actives dans les pratiques dévotionnelles. La colonne est ainsi, selon elle, centrale dans le dispositif émotionnel et sensoriel de la cérémonie d'auto-discipline. En effet, alors que de nombreux textes mentionnent l'extinction des bougies pendant l'administration de la flagellation ${ }^{45}$, la colonne était probablement la dernière chose que les fidèles voyaient avant d'entamer la flagellation rituelle, et la première qui leur apparaissait une fois les cierges rallumés ${ }^{46}$.

Le mobilier des oratoires s'inscrit souvent lui-même dans un cadre d'images que constituent les peintures murales. Celles de l'oratoire des disciplinati de San Rufinuccio à Assise ont fait l'objet de plusieurs études qui ont montré de fortes corrélations avec les laude chantées durant les offices, en particulier celle du vendredi saint, en particulier, l'une des plus célèbres, «Levate gl'occhie ${ }^{47}$. Exécuté vers 1348 par Puccio Capanna, et aujourd'hui conservé dans le musée de la cathédrale San Rufino à Assise, cet ensemble était composé en triptyque, d'une Crucifixion au centre, d'une Flagellation et d'une Déposition sur les côtés. On a ainsi souligné la pertinence de l'injonction du premier vers, "Levate gl' ochie ", qui conduit le fidèle à fixer son regard sur la fresque encadrant l'autel. Pendant que le texte énumère le traitement infligé au Christ lié à la colonne ${ }^{48}$, l'image a pour fonction de « supportare l'esecuzione delle laude ${ }^{49}$, au sens où elle sert de support à l'enclenchement d'un processus de représentation mentale.

Parmi les retables ayant formellement appartenu aux confréries flagellantes, il apparaît que la Flagellation est jusqu'au $\mathrm{Xv}^{\mathrm{e}}$ siècle environ, intégrée aux images narratives de la Passion, sur les volets ou dans la prédelle. En revanche, mentionnées par les textes, les tavole ou tavolette, portées parfois lors des processions ou disposées sur les autels, ou à côté, en guise de support pour la memoria, sont entièrement consacrées à la Flagellation. Or, dans ces petits panneaux apparaissent régulièrement des flagellants. S'il est fréquent de représenter les donateurs ou commanditaires en prière dans les images, il semble que ce procédé prend une dimension particulière dans le contexte de la dévotion flagellante, dont la pratique, appuyée par l'image, invite à reproduire les souffrances de la Passion. Plus qu'à une contemplation de l'image, c'est à une contemplation dans l'image que sont invités les devoti. Est ainsi mise en œuvre, par une forme d'enchâssement d'images, une relation spéculaire entre le Christ flagellé et les disciplinati qui se flagellent en mémoire et pour imiter la Passion du Christ.

\section{Des flagellants dans la Flagellation ou la mise en image du processus d'imitatio Christi}

21 Encouragée par saint Paul dans l'Épître aux Éphésiens, et les Pères de l'Église comme voie d'accès à Dieu, l'imitatio Christi voit sa nature et ses fins évoluer sous l'inflexion de la spiritualité des ordres mendiants ${ }^{50}$. Progressivement, les souffrances du Christ 
deviennent elles-mêmes un objet de dévotion et d'imitation. Aussi, la douleur physique et la souffrance sont des voies littérales pour imiter le Christ, et pour gagner le salut ${ }^{51}$, cependant que son sang devient célébré quant à lui comme source de vie et de purification. Ainsi, la pratique de la flagellation, longtemps confinée aux monastères, se répand chez les laïcs, fortement encouragée notamment par les dominicains, comme Venturino da Bergamo, Catherine de Sienne et Henri Suso ${ }^{52}$. Le succès est tel, dans les processions du XIII ${ }^{\mathrm{e}}$ siècle et parmi les mystiques, que l'Église condamne l'autodiscipline entraînant des effusions de sang ${ }^{53}$.

Dans les statuts des confréries, la flagellation est encadrée, parfois de manière très précise. Ceux de la Compagnia stretta della Madonna sancta Maria de la Vita di Bologna, datés de 1459, dans leur neuvième partie ("Quando si dibia andare a l'officio. El modo de dirlo e di fare disciplina », " quand il faut aller à l'office. Sur la manière de dire et de faire la disclipine»), sont riches d'enseignements: «Et d'abord est faite la confession suivant l'ordre écrit dans le livre IX, puis, l'office terminé, on se donne la paix et un ou deux des frères [...] commence la lauda $»^{54}$. Les lumières sont ensuite éteintes et le verset 14 du psaume 72 «Et fui flagellatus tota die, et castigatio mea in matutinis» («Et je suis frappé tout le jour, et chaque matin, je suis châtié ») donne le signal pour la discipline, cependant que la communauté récite le Miserere mei ; s'ensuit la récitation du De Profundis (Ps. 129). À la fin de la cérémonie, ayant dit quelques stances de la Passion, les membres se rhabillent rapidement et chacun va embrasser l'autel ${ }^{55}$. La discipline s'effectue au cours d'une cérémonie qui est une véritable expérience sensorielle, durant laquelle la lecture, la récitation, le chant, la contemplation de l'image et le geste sont des composantes essentielles ${ }^{56}$. L'interaction de ces éléments est la clé d'une mise en condition spirituelle et émotionnelle qui conduit à l'imitatio et à la dévotion.

Figure 9

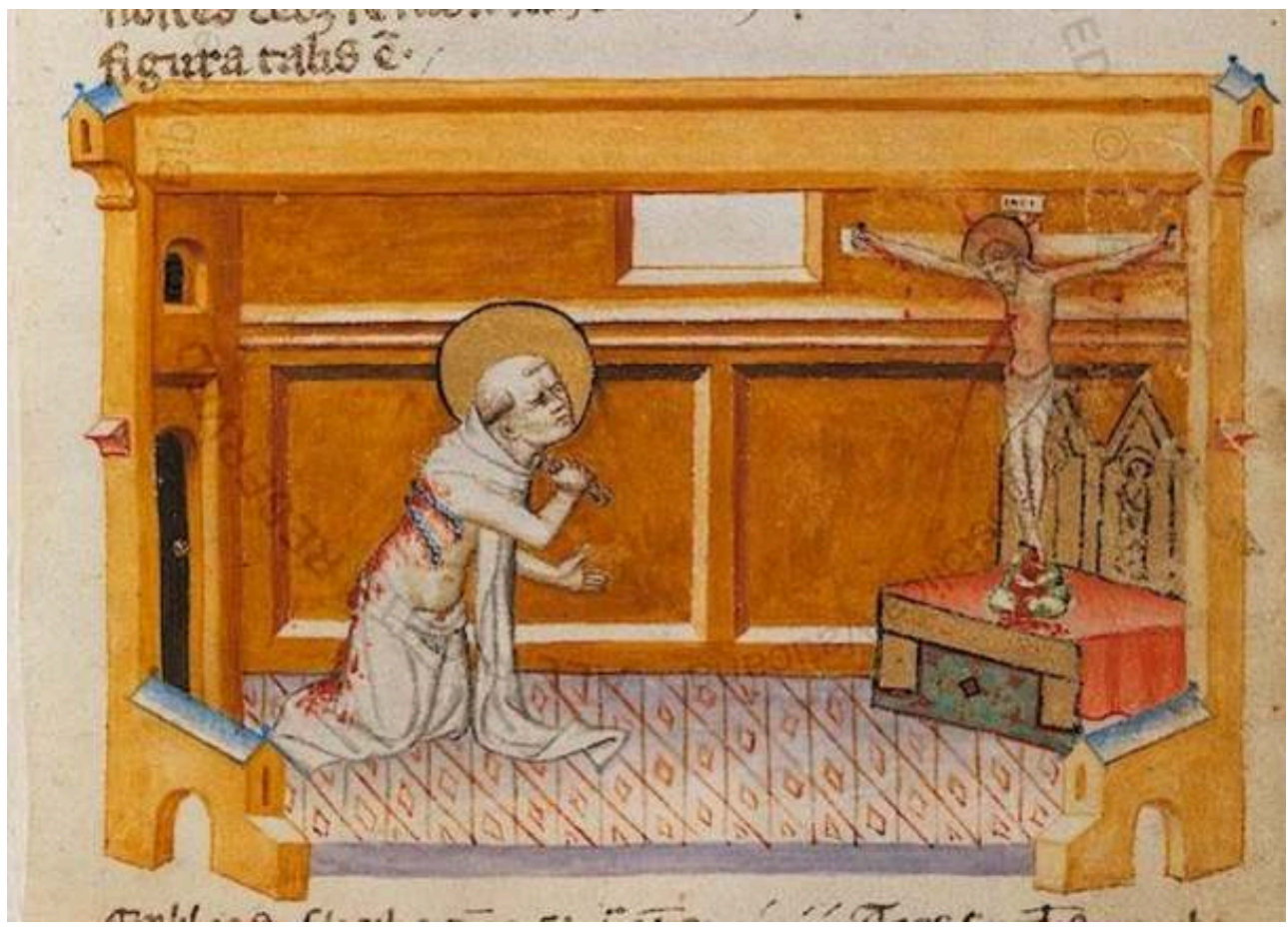

De modo orandi, début du xive siècle, peinture sur parchemin, Vatican, Bibliothèque apostolique vaticane, cod. Ross. 3. fol. 7r. 
illustrations des manuscrits du De modo orandi (De la manière de prier), reprenant les pratiques de prière de saint Dominique, éclairent quelque peu sur le rôle des gestes au moment de la prière, et sur la mise en condition pour la prière, par les gestes et par la contemplation de l'image ${ }^{57}$. En effet, l'imitatio passe par une projection mentale et une méditation sur les souffrances de Jésus, mais également par une mise en condition gestuelle, ainsi que le montre Jean-Claude Schmitt. L'auteur rappelle en effet qu'à partir du XII ${ }^{\mathrm{e}}$ siècle, le terme de gestus se voit préféré à celui, négativement connoté, de gesticulatio ${ }^{58}$. Glosant Hugues de Saint-Victor dans son De istitutione Noviciorum, l'auteur montre que désormais « les gestes manifestent à l'extérieur du corps (foris) la qualité de l'âme intérieure (intus) $»^{59}$. Ainsi, au XIII siècle, saint Dominique fait de la gestuelle corporelle une véritable pratique de prière, presque une condition. Toute l'attitude du corps doit permettre, sinon favoriser, un état mental disposé à la prière et à la contemplation, ou à l'humilité. Le De modo orandi, dont il existe trois manuscrits, consigne les différentes attitudes et gestuelles adoptées par saint Dominique lors de ses nombreuses séances de prière. Dans le codex Rossanus 3, datant du début du XIV ${ }^{\mathrm{e}}$ siècle (fig. 9 $)^{60}$, le saint est représenté dans une salle dépouillée, simplement pourvue d'un autel surmonté d'un triptyque sur lequel apparaît une figure nimbée, et d'un crucifix ${ }^{61}$. Dans ses prières, Dominique s'adresse à cette croix comme si le Christ était réellement présent, par l'image. D’ailleurs, le sang jaillissant de la plaie du Christ témoigne que la Passion est réellement en acte dans l'exercice spirituel du saint. Au folio $7 r$, ce dernier est représenté agenouillé, la chasuble remontée par-dessus les épaules, le torse et le dos nus. Le corps légèrement penché en avant, le sourcil froncé par l'intensité de la concentration, il se frappe le dos d'une double chaîne. De son dos intégralement meurtri et sanglant s'égoutte le sang qui imbibe sa tunique. La discipline, comme les autres modi orandi, permet de déclencher des états intérieurs d'humilité et de compassion, et de réactualiser les souffrances du Christ en les partageant et en les vivant. Le geste est donc ici un moyen qui, pour reprendre les termes de Jean-Claude Schmitt, "révèl[e une] double implication physique et spirituelle propre à l'état de dévotion ${ }^{62}$. Cette idée est précisément exprimée chez les Pères et les théologiens. Elle repose sur le postulat d'une interaction entre le mouvement du corps et celui de l'âme. C'est là la clé de la pratique de la discipline, qui est plus qu'une simple imitation. Elle déclenche le processus mental de prière et de dévotion. Celui-ci s'ancre en outre dans la contemplation de l'image, qui devient, comme le montrent les miniatures représentant saint Dominique, le second pôle nécessaire pour l'accomplissement du processus.

Cette considération nous conduit à interroger précisément le rôle de l'image dans ce contexte de l'administration de la discipline, et sur les moyens qu'elle déploie pour rendre possible l'imitatio. C'est chez le cistercien Aelred de Rievaulx (vers 1110-1166/67), qu'Olivier Boulnois trouve une théorie de l'image qui explicite les mécanismes mis en œuvre par ce support méditatif qu'est l'image. L'auteur reprend donc la définition que donne le moine cistercien de la méditation. Il s'agit, de façon traditionnelle, "d'une manière de rendre contemporain de la scène signifiée par le texte $»^{63}$. Mais ce résultat passe non plus par la prière ou par la contemplation (contemplatio), mais par une forme de "mise en présence » des personnages, des lieux, des scènes, à partir du texte. Ce processus qui consiste à rendre présent au fidèle est appelée par Aelred repraesentatio, « mise en présence ", donc.

«La base de la méditation aelrédienne, c'est la repraesentatio, la représentation par l'imagination de la scène de l'évangile considérée de sorte que celui qui médite participe activement à la scène ${ }^{64}$. 
Cette repraesentatio permet au lecteur de se projeter dans la scène narrée, au point de pouvoir y participer, par des gestes, des sentiments et des émotions.

Olivier Boulnois démontre ainsi que la re-présentation aelrédienne relève de l'expérience hallucinatoire et est un exercice visionnaire. Elle est donc intimement liée à une poétique du visuel et de l'image. Aelred de Rievaulx en prend pleinement la mesure et incite son lecteur à utiliser les images : "Sur ton autel, il suffira que tu aies une image du Sauveur pendant à la croix, qui te rendra présente (repraesentet) sa Passion pour que tu l'imites ${ }^{65}$. Cette actualisation perpétuellement possible par la méditation abolit toute historicité pour entrer dans un présent perpétuel qui est le temps du mystère et l'éternité divine. Dans le cas des confréries flagellantes, certaines images semblent la quintessence même de ce processus, d'autant plus qu'elles interviennent et deviennent efficaces lors de la mise en œuvre interactive d'un couple geste/image ${ }^{66}$.

\section{Figure 10}

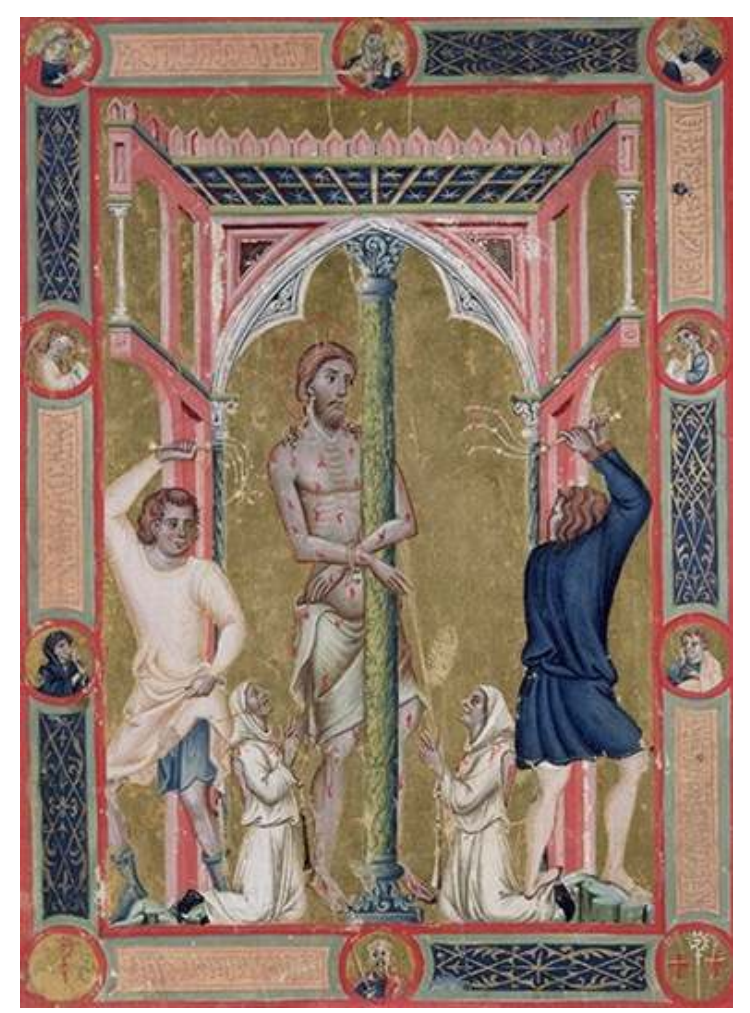

Luca di Paolo Veneziano (?), Mariegola de la Scuola di San Giovanni Evangelista de Venise, seconde moitié du xive siècle, tempera sur vélin, Paris, musée Marmottan, collection Wildenstein.

Citons par exemple la Flagellation du frontispice de la Mariegola de la Scuola di San Giovanni Evangelista de Venise, exécutée dans la seconde moitié du XIV siècle $^{67}$. Il s'agissait à l'origine d'une double page frontispice, mettant en regard une Deisis et une Flagellation ${ }^{68}$. Dans la première, le Christ et la Vierge trônant surplombent de petits pénitents, couverts de leur capuchon blanc, les mains jointes, en prière, le flagellum au poignet. À cette image visionnaire foisonnante et complexe, s'oppose une Flagellation beaucoup plus sobre (fig. 10). Seules cinq figures occupent l'espace : le Christ, au centre, s'étire sur les deux tiers de l'image, et domine de sa stature les deux bourreaux, plus petits et trapus. Dans le tiers inférieur, deux flagellants agenouillés prient en le 
regardant. Du fond d'or semble émerger une construction architecturée extrêmement allégée et aérienne, scandée par des modules tantôt rouges, tantôt bleus. La « maison de poupée ", structurée par une perspective centrale soulignée par un plafond à caissons bleus et or, se termine par une baie trilobée, au centre de laquelle se dresse la colonne marmoréenne de la Flagellation. Le cadre architectural est repris et souligné par le cadre peint de la page enluminée. Se succèdent en effet des bandes rouges et bleues, agrémentées de rinceaux dorés, ou de caractères pseudo-koufiques. L'ensemble est ponctué de dix médaillons dans lesquels figurent anges, prophètes, et saint Paul, figurant le lien entre ancienne et nouvelle Lois. Le cadre est clos par l'emblème de la confrérie, inscrivant cette dernière dans la continuité de la mémoire de la Passion. Plus encore, la re-présentation de la Passion est ici littéralement mise en image par la création d'une « métalepse » visuelle, entendue comme un jeu permanent d'inclusion et d'exclusion de deux niveaux d'images ${ }^{69}$. Ici, le processus s'opère au sein du cadre extérieur, qui forme lui-même un premier « degré » d'image. Nous sommes en effet en présence de deux niveaux d'images - si l'on exclut temporairement le cadre - dont l'une semble être l'émanation de l'autre. Le premier voile d'image est constitué par les deux petits flagellants, de proportion nettement inférieure aux trois autres figures. Ils sont tous deux en prière, agenouillés autour de la colonne. Le second voile (ou niveau) d'image est composé de Jésus et des deux bourreaux. Ils font référence à une temporalité historique différente de la contemporanéité des flagellants, différence relayée par le contraste entre la taille et le volume des différentes figures. La haute stature des trois figures est renforcée par la composition pyramidale qui guide le regard et exclut totalement les deux pénitents au bas de l'image: au triangle que forment les trois chevelures blond vénitien, prolongé par l'arc trilobé où culmine le chapiteau de la colonne du supplice, s'ajoute le jeu de regards entre Jésus et les deux sbires. Jésus fixe en effet le bourreau à droite de l'image qui, bien que de dos, semble lui rendre son regard, cependant que le bourreau de gauche lance un coup d'œil latéral vers son homologue. Cette triangulation des regards fonctionne en vase clos et exclut les petits pénitents. Pourtant, le blanc de leurs vêtements est rappelé par l'arcade de l'édifice, ce qui ouvre le regard et lui permet une circulation de bas en haut. Ainsi, sur le triangle des regards au centre de l'image, se surimpose une circulation en cercle des cinq chefs pris dans leur ensemble. Les lignes formées par les regards appuyés des flagellants en direction du Christ qui les domine, interrompent le cercle central, renforçant ainsi l'inclusion du premier niveau d'image dans le second.

Ce jeu de glissement, qui nous semble permettre l'emploi du terme de métalepse, est également créé par la construction extrêmement complexe de l'espace, contrairement à l'impression première d'un effort malhabile de perspective centrale, venu échouer sur un fond d'or. Au contraire, "l'échec" de cette construction est précisément préparé. Au premier plan, semblant sur le point de déborder du cadre, les petits flagellants font office d'admoniteurs. En haut de l'image, l'architecture creuse au contraire l'espace en profondeur, pour créer un écrin à la Flagellation proprement dite. Or, les bourreaux, légèrement plus en retrait du seuil que les pénitents, relèvent en réalité d'un espace différent : ils sont montés sur de petits édicules rocheux, rappelant les représentations du Golgotha des Crucifixions. Les deux bourreaux seraient donc dans une forme d'extérieur, qui se surimpose visuellement, de manière littérale, à l'espace des flagellants: le bourreau de droite «écrase » les mollets du flagellant en prière. Le Christ, quant à lui, alors même qu'il est abrité par le porte-à-faux de l'édifice, clé dans l'ancrage perspectif, flotte paradoxalement au-dessus du sol, les pieds dans le 
vide. Ce jeu de glissement entre deux espaces est enfin prolongé, ou plutôt créé par la colonne, élément central de l'image, dont la lecture, du bas vers le haut met en œuvre un jeu d'optique : le bas de la colonne est, comme les flagellants, au premier plan et en bordure de cadre, assumant une bi-dimensionnalité frontale. Mais le chapiteau, en haut de l'image, se loge sous l'arc brisé, c'est-à-dire dans la profondeur du champ ménagé par la structure architecturale. Il ne s'agit en aucun cas de maladresse de la part de l'artiste: il n'aurait pas été difficile de prolonger le fût de la colonne pour que le chapiteau vienne se plaquer sur le devant de l'édifice, et non dans le fond. Il y a donc bien glissement d'un espace à un autre, de l'image des flagellants à celle de la flagellation, séparés par divers procédés, mais réunis par la colonne. Cette composition sophistiquée est en réalité une mise en image du processus d'imitation en œuvre dans la dévotion flagellante, ou plus précisément son résultat. La Flagellation est ici comme une projection visionnaire des pénitents, la représentation d'une image mentale, comme celles sollicitées par les prédicateurs ou les textes dévotionnels contemporains ${ }^{70}$. Ici, les membres de la confrérie ne sont pas en train de s'administrer la discipline. En revanche, celle-ci a déjà eu lieu, comme en témoignent les plaies sanglantes visibles dans les larges encoches ménagées sur leur vêtement, et les flagella au poignet ou à la taille. Il s'agit ici de l'étape suivante, celui de la dévotion, durant laquelle est réactualisée la Passion, re-présentée, en pensée, en acte, et ici en image. Les plaies du Christ sont celles des flagellants, et réciproquement. Les coulures rouges se confondent également avec la crosse, emblème de la confrérie, peinte sur le torse et les épaules des confrères. Cette Flagellation-frontispice se présente donc comme un feuilletage de sens qui lui confère une signifiance très riche. Elle justifie notamment l'existence même de la confrérie, en l'insérant dans la mémoire de la Passion et présente aux novices, une représentation active de l'imitatio. 


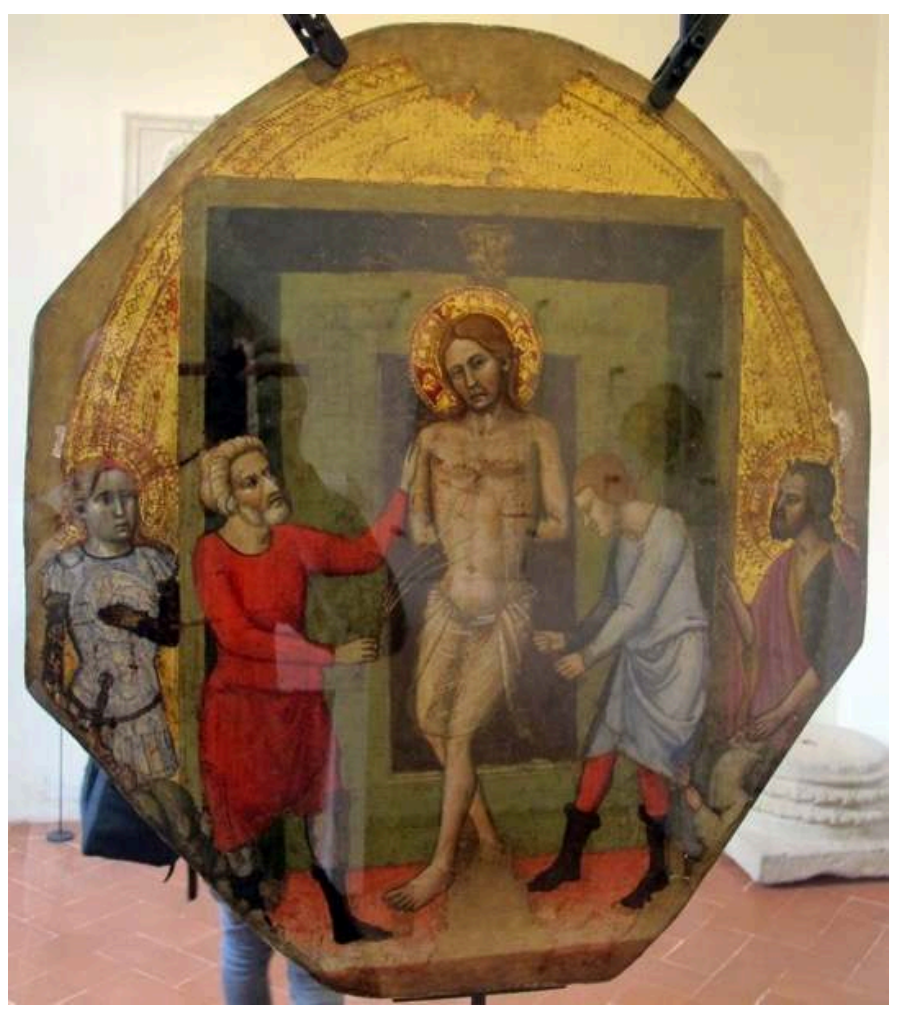

Jacopo di Michele, étendard processionnel avec Flagellation, vers 1370-1390, tempera sur bois, 120×132 $\mathrm{cm}$, Pise, San Miniato, museo d'Arte Sacra.

On retrouve cette même structure d'enchâssement dans d'autres images, notamment des panneaux, comme l'étendard peint par un suiveur de Giovanni di Nicola, daté du $\mathrm{XIV}^{\mathrm{e}}$ siècle $^{71}$, ou ceux de Jacopo di Michele, l'un exécuté vers $1370-1390^{72}$ pour la confrérie des Battuti di san Giovanni Battista de Montopoli (fig. 11), et l'autre, retouché au $\mathrm{XV}^{\mathrm{e}}$ siècle, appartenant à une collection privée $\mathrm{e}^{73}$. La composition des trois panneaux est strictement identique : la Flagellation proprement dite, se déroule au centre de l'image, au troisième plan, dans un édifice ouvert, construit selon une perspective centrale, à la manière d'une maison de poupée. La pièce est donc artificiellement ouverte sur l'extérieur dont elle se démarque par une petite marche-seuil. À l'extérieur, relégués au premier plan et sur les côtés, de petits pénitents dissimulés par leur capuchon, se tiennent agenouillés, en prière. À leur côté, dans chaque image, se tiennent des saints admoniteurs. Ici, les membres des confréries sont exclus de l'espace de la scène biblique. Ils l'observent depuis l'extérieur, et leur regard, comme le nôtre, est invité à cette contemplation par les figures saintes qui les accompagnent. Dans les trois cas, l'admoniteur se situe à droite de l'image et pointe Jésus de l'index. Aucun des membres n'est en train de se donner la discipline, mais celle-ci est explicitement rappelée par les dos sanglants qui ponctuent linéairement le premier plan $^{74}$. Le jeu d'imbrication des espaces recrée la différence de nature des différentes figures: les minuscules flagellants sont maintenus à l'extérieur, de même que les intercesseurs; mais la taille de ces derniers, aussi importante que celle du Christ, leur position et leur geste en font des figures de transition et de seuil vers la scène qu'il faut contempler. Pour le spectateur réel qui va se livrer à la discipline, il s'agit moins d'une Flagellation que de flagellants devant une Flagellation. C'est l'efficace de l'image qui est ici illustrée, en ce 
que la Flagellation, isolée dans la maison de poupée, fonctionne encore une fois comme la projection mentale des pénitents en prière. Ils en sont la cause première, l'origine. Ces panneaux mettent en scène, re-présentent le processus d'imitatio et son résultat: l'identification au Christ. Cette identification se traduit visuellement, et physiquement par le sang et par la plaie.

Ces flagellants qui se flagellent aux pieds du Christ sont donc plus que des admoniteurs. Ils sont des modèles, des guides vers l'imitatio et des miroirs. Et leur image dans l'image fait de ces panneaux de dévotion une re-présentation perfomative de l'imitatio. En premier lieu, ces images sont en effet capables d'engager le spectateur dans une performance ${ }^{75}$. Mais elles sont également performatives au sens où les moyens mis en œuvre dans la représentation - le tableau, pour reprendre le terme de Bertrand Rougé -, permettent de réaliser, de rendre réelle l'historia, c'est-à-dire le contenu figuré de l'image ${ }^{76}$. Dans les derniers exemples présentés, l'enchâssement d'images par la mise en abyme ou par la métalepse spatiale créée un espace-temps qui échappe à la temporalité humaine, et qui est celui du mystère. Ainsi, l'image rejoue elle aussi l'expérience dévotionnelle en train de s'accomplir devant et par elle, par les flagellants.

\section{NOTES}

1. André vaUCHEZ, Les laïcs au Moyen Âge. Pratiques et expériences religieuses, Paris, Éditions du Cerf, 1987 ; Dominique RIGAUX, Daniel RUSSO, Catherine VINCENT (éds), Expériences religieuses et chemins de perfection dans l'Occident médiéval. Études offertes à André Vauchez par ses élèves, Paris, De Boccard, 2012.

2. John HENDERSON, Piety and Charity in Renaissance Florence, Chicago, Londres, University of Chicago Press, 1997. Les distinctions s'avèrent toutefois fluctuantes. Sur le mouvement confraternel, voir Gilles Gerard MEERSSEMAN, Ordo fraternitatis, Confraternite e pietà dei laici nel Medioevo, Rome, Herder, 1977, 3 vols; Ronald F. E. WEISSMAN, Ritual Brotherhood in Renaissance Florence, New York, Londres, Paris, Academic Press, 1982 ; Le mouvement confraternel au Moyen Âge : France, Italie, Suisse, actes de la table ronde Lausanne, 9-11 mai, 1985, Rome, École française de Rome, 1987 ; A. VAUCHEZ, Les laïcs au Moyen Âge, op.cit.; Marina GAZZINI (dir.), Studi confraternitali: orientamenti, problemi, testimonianze, Florence, University Press, 2009 ; ead. (dir.), «Bibliografia medievistica di storia confraternale ", Reti Medievali Rivista, vol. V, n²1 2004, [en ligne : <http://www.rmojs.unina.it/ index.php/rm/article/view/4534/5076 >]

3. Catherine VINCENT, "Discipliner du corps et de l'esprit chez les Flagellants au Moyen Âge ", Revue historique, t. 302, fasc. 3, juillet/septembre 2000, p. 593-614, ici p. 605. Voir également Jean LECLERCQ, « La flagellazione volontaria nella tradizione spirituale dell'occidente », dans Ludovico SCARAMUCCI (dir.), Il movimento dei disciplinati nel settimo centenario dal suo inizio, Convegno internazionale, Perugia, 25-28 settembre 1960, Pérouse, Deputazione di Storia patria, 1962-1965, p. 73-83, ici p. 79.

4. C. VINCENT, « Discipliner du corps et de l'esprit », op. cit., p. 597.

5. Ibid., p. 605.

6. Ibid., p. 596 ; Patrick VANDERMEERSCH, La chair de la Passion : une histoire de foi. La flagellation, Paris, Éditions du Cerf, 2002, p. 115-117. 
7. Jean WIRTH, L'image à la fin du Moyen Âge, Paris, Éditions du Cerf, 2011, p. 330-334.

8. Hans Belting, Image et culte: une histoire de l'image avant l'époque de l'art, trad. de l'allemand par Frank Muller, Paris, Éditions du Cerf, 1998 ; Paolo sANVITo, Imitatio : l'amore dell'immagine sacra. Il sentimento devoto nelle scene dell'imitazione di Cristo, Pescara, Ed. ZIP, 2009, p. 12-15.

9. Giovanni CECCHINI, "Raniero Fasani et les Flagellants ", Mélanges de L'École française de Rome, 87-1, Rome, École française de Rome, 1975, p. 339-352.

10. Les confréries flagellantes ont nourri une littérature très abondante. Nous ne citons que quelques références: L. SCARAMUCCI (dir.), Il movimento dei disciplinati, op.cit.; G. G. MEERSSEMAN, Ordo fraternitatis, op. cit; John HENDERSON, «The flagellant movement and flagellant confraternities in Central Italy, 1260-1400», Studies in Church History, vol. 15, 1978, p. 147-160.

11. Le passage est demeuré célèbre et est fréquemment cité: «Durant l'année précédente, comme l'Italie tout entière était souillée de scandales et de turpitudes, un certain mal, subit et jusque-là inconnu, s'en prit d'abord aux habitants de Pérouse, puis de Rome, et enfin à presque tous les peuples d'Italie. C'est pourquoi une telle crainte du Seigneur se déversa sur eux, que nobles et vilains à égalité, jeunes et vieux, même les enfants de cinq ans, allèrent à travers les places publiques, nus à l'exception des parties honteuses, toute vergogne oubliée, deux par deux en procession, chacun tenant en ses mains un fouet de plusieurs lanières et avec force gémissement et lamentation, se frappant par-dessus les épaules, jusqu'à ce que le sang coule ». Nous traduisons ( $\mathrm{Sub}$ precedenti anni circulo, cum tota Italia multis esset flagitiis et scleribus inquinata, quedam subitanea compunctio et a seculis inaudita invasit primitus Perusinos, Romanos postmodum, et deinde fere Italie populos universos. In tantum itaque timor Domini irruit super eos, quod nobiles pariter et ignobiles, senes et iuvenes, infantes etiam quinque annorum, nudi per plateas civitatum, opertis tantum pudendis, deposita verecundia, bini et bini processionabiliter incedebant; singuli flagellum de corigiis in manibus habentes et cum gemitu et ploratu se acriter super scapulis usque ad effusionem sanguinis verberantes [...]» (Anonyme padouan, Chronicon Marchiae Tarvisiniae et Lombardiae, dans Rerum italicarum sciptores, Editio altera, op. cit., VIII, III, 44-45, cité par G. G. MEERSSEMAN, Ordo fraternitatis, op. cit., vol. 1, p. 458. Voir d'autres exemples p. 458-461.)

12. "Non solum in die, verum etiam in nocte, cum cereis accensis, [...] centeni et milleni ac quoque decem millia per civitates et ecclesias circuibant, et se ante altaria humiliter prosternebant, precedentibus eos sacerdotibus cum crucibus et vexillis ", ibid.

13. Salimbene DE ADAM, Cronica, Giuseppe SCALIA (éd.), Turnhout, Brepols, 1999, I, 1. 1250-1287.

14. Giovanni SerCAmBI, Chronique, Lucques, Biblioteca Capitolare, ms. 108, fol. 618.

15. La discipline pouvait être administrée par des petites branches souples de bouleau ou des lanières de cuir, parfois complétées par des nœuds, des osselets, des boules de plomb ou des pointes de fer (C. VINCENT, « Discipliner du corps et de l'esprit », op. cit., p. 601).

16. Giovanni VILlanI, Cronica, Rome, Bibliothèque apostolique vaticane, L.V.III, 296.

17. «Aujourd'hui, le 19 avril 1377, au matin, chacune des compagnies de flagellants a paradé à travers Florence, avec de nombreuses bannières et tavole de Notre Dame, de San Giglio (Egidio), et de nombreux crucifix et tavole et étendards des compagnies ", Anonyme florentin, Diario d'anonimo fiorentino, dall'anno 1358 al 1389, dans Cronache dei secoli XIII-XIV, Marco TABARINI (éd.), Florence, 1876, p. 331. Nous traduisons les sources italiennes de cet article.

18. Sur les statuts de manière générale, voir Giuseppina De SANDRE GASPARINI, Statuti di confraternite religiose di Padova nel Medio evo, Padoue, Istituto per la storia ecclesiastica padovana, 1974 ; Lia SBRIzIOLO, «Per la storia delle confraternite veneziane : dalle deliberazioni miste (1310-1476) del Consiglio dei dieci. Le scuole dei battuti », dans Miscellanea Gilles Gerard Meersseman, II, Padoue, Atenore, 1970, p. 715-763 ; Anna ESPOSITO, «Statuti confraternali italiani del tardo Medioevo. Aspetti religiosi e comportamentali ", dans Gisela DROSSBACH (dir.), Von der Ordnung zur Norm: Statuten in Mittelalter unf Früher Neuzeit, Munich, Vienne, Zurich, Ferdinand Schöningh, 2009, p. 297-309. 
19. "Quod homines dicte congregationis debeant reverire picturas sanctorum cum fuerint coram eis. Item statuimus et ordinamus quod semper, quando aliquis de congregatione fuerint coram pictura Domini Nostro Ihesu Christi et beate Marie virginis matris eius, in eorum reveranciam debeat sibi inclinare", Statuto dei Disciplinati del Borgo Porta Nova di Vicenza (1263), § 8, cité par G. G. MEERSSEMAN, Ordo fraternitatis, op. cit., p. 480.

20. Ibid., p. 482, § 12 .

21. Ibid., p. 481, § 24. « Nous statuons et ordonnons que quand l'un des membres de la fraternité se trouve devant une peinture ou une image de notre Seigneur Jésus-Christ crucifié et de la glorieuse Vierge Marie, il doive incliner le chef avec révérence " (Item statuimus et ordinamus quod quando aliquis de fratalia fuerit coram pictura seu ymagine domini notri Yhesu Christi crucifissi et gloriose virginis Marie, debeat cum reverentia caput inclinare »).

22. Sur les pratiques de la prière et notamment l'interrelation entre les gestes, les images et les mots, voir Jean-Claude SchmiT, La raison des gestes dans l'Occident médiéval, Paris, Gallimard, 1990 et Éric PALAzZo, Peindre c'est prier : anthropologie de la prière chrétienne, Paris, Éditions du Cerf, 2016. Ce dernier consacre un chapitre à l'étude du crucifix et du mobilier liturgique dans le processus dévotionnel. Nous reviendrons sur ce point.

23. « (La confraternità) deve avere inoltre un'insegna di Cristo bella e devota da portare in procesisone e un sigillo con al centro Cristo flagellato ed attorno la leggenda della compagnia ", Confraternita dei Disciplinati di S. Nicola di S. Francesco, Palerme, Biblioteca Centrale Regionale di Palermo, I F 3.

24. Libro delle memorie. Ricordi, brevi pontificij, partiti, e narrazione delle cose piu rimarcabili della Compagnia de' Battuti di S. Maria della Neve, o del Confalone di Modena, Modène, Biblioteca Estense, Campori 161 (=y.I.4.21).

25. « Nello Stendardo [...] contemplavasi l'immagine del nostro Salvator Giesu Cristo flagellato alla Colonna, in memoria della sua acerbissima passione, percio la Compagnia del Cristo flagellato appellavasi ", Libro delle memorie, op. cit., fol. 30.

26. "E in questo luogo tenghino una imagine di cristo", Florence, Archivio di Stato, Capitoli delle compagnie religiose soppresse da Pietro Leopoldo, 136, chapitre II, fol. 4-5v).

27. "Il est ordonné que cette compagnie se rassemble [...] et qu'en ce lieu soit conservée une image du Christ [...]. Chaque vendredi, les membres doivent se réunir dans ce lieu. Ils doivent se tenir en silence [...] Et quand ils sont vêtus et que l'un d'eux se tienne devant l'image du Christ, les mains fermées et levées et se signant et dise alors ce qui est écrit en rouge : "Notre secours est dans le nom de Dieu qui a fait le ciel et la terre ». Et tous les autres doivent lever leurs mains jointes et répondre "Amen ». Puis ils doivent prendre les disciplines et celui qui est au centre doit dire « Emparez-vous de la discipline, de peur qu'Il ne s'irrite et que vous ne périssiez dans votre voie» («E ordinato che questia compagnia si devva raunare [...] e in questo luogo tenghino una imagnie di cristo [...] Et ciascuno venardi si venghino alluogho ordinato tutti quanti. Et ive tenghino silençio [...]. E quando sono vestiti e uno di loro stia dinançi alla imagine di cristo tenendo le mani chiuse e alte $e$ segnandosi e poi dicha questo che e scritto di ginabro. Adiutoriom nostrum in nomine domini qui fecit celum et terram. E tutti gli altri lievino le mani alte e giunte e rispondino e dichino Amen. E poi lievino le discipline di terra. E poi quello medesimo che disse [...] dica chosi Adprehendite disciplinam ne quando irascatur dominus, ne pereatis de via gratias »).

28. Pierre-Jean AUGIER, Psaumes nouvellement traduits sur l'hébreu et mis dans leur ordre naturel avec des explications et des notes critiques, Paris, 1809, vol. 3, p. 7.

29. Venise, Fondation Cini, inv. 2041. Voir Lyle HuMPhreY, The Illumination of Confraternity and Guild Statutes in Venice, ca. 1260-1500: Mariegole Production, Iconography, and Use, PhD, New York University, Institute of Fine Arts, 2007, cat. 10.3.

30. L'illustration de la règle de 1418-1422 de la même confrérie de San Giovanni Evangelista à Venise (Boston, Boston Public Library, ms. Med.147), reprend, quelques années après la première, une composition identique, à ceci près que l'étendard a été placé à côté de l'autel. Voir L. HUMPHREY, The Illumination of Confraternity, op. cit., cat. 33.2c. 
31. Venise, BNM, cod. lat. II, 119, fol. 1R ; L. HUMPHREY, The Illumination of the Confraternity, op. cit., pl. 19d.

32. Modène, Bibliothèque Estense, ms. 2, dernier quart du XIV ${ }^{e}$ siècle. Cité par Andreas DEHMER, «Passio und Compassio : Geisselungsrituale italienischer Bussbruderschaften», dans Jan F. VAN DIJKHUIZEN et Karl A. E. ENENKEL, The Sense of Suffering : Constructions of Physical Pain in Early Modern Culture, Leyde, Boston, Brill, 2009, p. 221-252 ; id., « Mobile Passionsdarstellungen als Leitbilder kollektiver Geisselungsrituale im spätmittelalterlichen Italien », Das Münster 55, 2002, p. 200-207.

33. Exemple parmi d'autres, les inventaires de l'une des plus puissantes confréries flagellantes de Florence, la Compagnia di Gesù Pellegrino, témoignent de la grande variété des images dont disposaient ses membres. Katheen Giles ARTHUR, "Cult Objects and Artistic Patronage of the Fourteenth Century Flagellant Confraternity of Gesù Pellegrino ", dans Timothy VERDON (dir), Christianity and the Renaissance, image and religious imagination in the Quattrocento, Syracuse (N. Y.), Syracuse University Press, 1990, p. 336-360 ; voir également Ludovica SEBREGONDI, «Religious Furnishings and Devotional objects in Renaissance Florentine Confraternities ", dans Konrad EISENBICHLER (dir), Crossing the Boundaries, Christian Piety and the Arts in Italian Medieval and Renaissance confraternities, Kalamazoo, Medieval Institute Publications, Western Michigan University, 1991, p. 141-160.

34. «I Flagellanti, Battuti o Disciplinati scelsero d'ordinario, come distintivo o emblema l'immagine di Gesù flagellato, oppure Crocifisso, e talvolta l'insegna della croce accompagnata dagli strumenti della Passione ", (Giacomo Carlo BASCAPÉ, «I sigilli delle confraternite », dans L. SCARAMUCCI (dir.), Il movimento dei disciplinati, op. cit. p. 309-315, ici p. 309).

35. Ibid.

36. Ibid.

37. Statuts de la Scuola di San Marco, Venise, milieu du XIV siècle, Venise, BMC, ms. IV. fol. 83.

38. Edward B. GARRISON, Italian Romanesque Panel Painting, An illustrated Index, Florence, Olschki, 1949, n. 481 ; Luciano BELLosI, La pecora di Giotto, Turin, Einaudi, 1985, p. 133, 157 ; Filippo TODINI, La pittura umbra dal Duecento al primo Cinquecento, Milan, Longanesi, 1989, p. 123.

39. Si elle est inédite pour les croix processionnelles, cette combinaison iconographique est très fréquente sur les panneaux ou étendards.

40. Spinello Aretino, étendard processionnel, vers 1395 (New York, Metropolitan Museum of Art, inv. 13. 175). Voir également l'ensemble de panneaux textiles de Niccolò di Pietro Gerini, exécutés vers 1390. Longtemps conservé dans l'église de Withyham en Grande-Bretagne, ces scènes de la Passion ont été vendues par Southeby's en 2012 et ont rejoint une collection privée (Voir Caroline VILLERS, "Painting on canvas in Fourteenth Century Italy ", Zeitschrift für Kunstgeschichte, 58, 1995, p. 338-358).

41. Exécuté entre 1445 et 1458 pour la Compagnia del Santissimo Sacramento, à Sienne. Conservé à San Giovanni d'Asso, Piccolo Museo della Compagnia del Santissimo Sacramento.

42. Sur l'iconographie du Vir dolorum, voir Erwin PANOFSKY, «Imago Pietatis: Ein Beitrag zur Typengeschichte des 'Schmerzensmanns' und der 'Maria Mediatrix'», Festschrift für Max J. Friedländer zum 60 Geburtstage, Leipzig, E. A. Seemann, 1927, p. 261-308, réédité dans id., Peinture et dévotion en Europe du Nord à la fin du Moyen Âge, Paris, Flammarion, 1997 ; Sixten RINGBOM, De l'icône à la scène narrative, trad. de l'anglais par Patrick Joly, Laurent MILÉsI, Paris, Gérard Monfort, 1997 ( $1^{\mathrm{e}}$ éd. Abo, 1965); Hans Belting, L'image et son public au Moyen Âge, trad. de l'allemand par Fortunato Israël, Paris, Gérard Monfort, (1e éd. Berlin, 1981).

43. Cité par K. G. ARTHUR, «Cult Objects », op. cit, p. 350. (Florence, Archivio di Stato, Compagnie Religiose Soppresse $910, n^{\circ} 6$, fol. 93, $n^{\circ} 98$ ). La même année, la compagnie acquiert une bannière peinte représentant la Flagellation, destinée à être présentée devant le retable d'autel.

44. Florence, Archivio di Stato, Compagnie Religiose Soppresse 910, $n^{\circ} 6$, fol. 90v et $91 \mathrm{r}$. 
45. Pour la Compagnia Gesù Pellegrino, voir Florence, Biblioteca nazionale centrale, Cod. Magl. VIII. 1282, fol. 58-73.

46. Nous suivons ici le raisonnement de K. G. ARTHUR, «Cult Objects », op.cit., p. 350.

47. Pietro SCARPELLINI, «Echi della lauda nella pittura umbra del XIII e XIV secolo», dans Enrico MENESTò (dir.) Le laude drammatiche umbre delle origini, Atti del V Congresso internazionale, Viterbe, Centro di studi sul teatro medioevale e rinascimentale, 1981 p. 165-185 ; Luigi ALLEGRI, Teatro e spettacolo nel Medioevo, Bari, Laterza, 1988, p. 214.

48. L'un des récitants, notamment, prenant le rôle de Marie Madeleine, énumère les tourments et humiliations de la Passion: "Puoi che Cristo àver legato/ Comenzarlo a tormentare, nello volto fu sputato, e già non se potea nettare/ quille carni preziose/ da li sputi obrubriose » (Vincenzo de BARTHOLOMAeIs, Laude drammatiche e rappresentazioni sacre, Florence, Le Monnier, 1943, vol.1, p. 317 , sq.

49. Mara NERBANO, «'Levate gl'ochie e resguardate'. La drammaturgia della Passione tra meditazione e pratiche sceniche", Annali dell'Università di Ferrara, Sezione Storia, vol. 2, 2005, p. 13-48, ici, p. 16.

50. P. SANVITO, Imitatio, L'amore dell'immagine sacra, op. cit.

51. Carolyn w. BYnUM, Holy Feast, Holy Fast: The religious Significance of Food to Medieval Women, Berkeley, University of California Press, 1988; Giles CONSTABLE, "The Ideal of the Imitation of Christ ", dans Id., Three Studies in Medieval and Religious Thought, Cambridge, Cambridge University Press, 1995 ; Niklaus LARGIER, In Praise of the Whip :A Cultural History of Arousal, trad. de l'allemand par Graham Harman, New York, Zone Books, 2007 ; Gabòr KLANICZAY, « Illness, self-inflicteed body pain and supernatural stigmata : three ways of identification with the suffering body of Christ ", dans Christian KöTZL, Katariina MUSTAKallio, Jenni KuUliala, (dirs) Infirmity in Antiquity and the Middle Ages, Social and Cultural Approaches to Health, Weakness and Care, Londres, New York, Routledge, Taylor \& Francis group, 2016, p. 119-136.

52. Jean LECLERCQ, « La flagellazione volontaria nella tradizione spirituale dell'Occidente », dans Il movimento dei disciplinati, op. cit., p. 73-83 ; Gabòr KLANICZAY, «Illness, Self-inflicted Body Pain », op. cit., p. 133-134.

53. P. VANDERMEERSCH, La chair de la Passion, op. cit. p. 89.

54. «Et in prima fata la confessione secundo l'ordine scrito in lo nostro libro novo, e finito l'officio dagase la paxe e uno o dui fradelli [...] comença la laude», Giuseppe ALBERGIO, "Contributi alla storia delle confraternite dei disciplinati », op. cit., p. 156-252, ici p. 217.

55. «E consequente digase quelache stancie de passione e incontinenti se vestano », Ibid.

56. Mara NERBANO, "Confraternite disciplinate e spazi della devozione", dans Adriano PROSPERI, Nicholas TERPSTRA, Stefania PASTORE (dirs), Brotherhood and Boundaries/ Fraternità e Barriere, Pise, Edizioni della Normale, 2011, p. 31-49. Voir également Éric PALAzzo, L'invention chrétienne des cinq sens dans la liturgie et l'art du Moyen Âge, Paris, Éditions du Cerf, 2014 ; id., Peindre c'est prier, op.cit.

57. Dominique lui-même désire le martyre, voie de l'imitation du Christ et partant, de la rédemption. À des hérétiques qui le conspuent et qui s'étonnent de la joie sereine qu'il affiche, il répond: «Je vous aurais prié de ne pas me faire mourir tout de suite par des coups subits, mais de mutiler chacun de mes membres, petit à petit, et l'un après l'autre, ensuite de placer sous mes yeux les morceaux de mes membres que vous auriez coupés puis de m'arracher aussi les yeux, et en dernier lieu de laisser mon corps, mis en pièces et à demi-mort, se vautrer dans son propre sang" " "Rogassem uos ne repentinis me subito perimeretis uulneribus, sed paulatim et successiue membra singula mutilantes, deinde ostensis coram meis oculis detruncatis membrorum particulis, ipsos etiam oculos eruentes ad ultimum semiuiuum laceratumque corpus sic permitteretis in suo sanguine uolutari, uel prorsus ad libitum necaretis ", JACQUES DE VORAGINE, La légende dorée, chap. 109, traduction A. Boureau, Paris, Gallimard, 2004, p. 585, texte latin ed. Maggioni, p. 721. 
58. Jean-Claude sснмітт, «Entre le texte et l'image : les gestes de la prière de saint Dominique », dans Richard TREXLER (dir.), Persons in Groups, Social Behavior as Identity Formation in Medieval and Renaissance Europe, Binghamton, New York, Center of medieval and early Renaissance studies, 1985, p. 195-220.

59. J.-Cl. Schmitt, « Entre le texte et l'image », op. cit, p. 196.

60. De modo orandi, début du $\mathrm{XIV}^{\mathrm{e}}$ siècle, peinture sur parchemin, Vatican, Bibliothèque apostolique vaticane, cod. Ross. 3. fol. 7r., <https://digi.vatlib.it/view/MSS_Ross.3 >

61. É. PALAZzo, Peindre c'est prier, op.cit., chapitre 4 « Les neuf modes de prière de saint Dominique. Un traité eucharistique en image ».

62. J.-Cl. sснмітт, "Entre le texte et l'image », op. cit, p. 202. Pour l'auteur, l'usage de la langue vulgaire et la présence des images accroît le degré de proximité et d'intimité entre les frères et le saint, « le regard qu(e chacun) posait sur l'illustration lui permettant déjà de participer à son extraordinaire dévotion, avant même de s'essayer à la reproduire dans son propre corps ", (p. 209).

63. Olivier BoulnoIs, Au-delà de l'image. Une archéologie du visuel au Moyen Âge, Ve-XIVe siècle, Paris, Seuil, 2008, p. 124 et sq. L'auteur s'appuie sur les sermons du cistercien, ainsi que sur l'opuscule La vie de recluse.

64. Ibid., p. 125.

65. Aelred DE RIEVAULX, La vie recluse, § 26, cité par O. BOULNOIS, Au-delà de l'image, op. cit, p. 128.

66. «La représentation mentale constitue le noyau primordial permettant une visualisation et une intimité, puis vient la représentation liturgique qui permet l'imitation du Christ à partir de signes, de gestes visibles ; enfin, même pour celui qui sait lire, la mémoire sera soutenue par un objet extérieur, la ressemblance des images visibles ». Ibid., p. 129.

67. L. HUMPHREY, The Illumination, op. cit., cat. 10.

68. La première est conservée au Museum of Art de Cleveland (inv. 59.128), la seconde à Paris, au musée Marmottan (collection Wildenstein, M. 6098).

69. Ce terme nous a été suggéré par Stéphanie Wyler. Dans son Encyclopédie, César Chesneau Dumarsais définit la métalepse en « [...] une sorte de métonymie, par laquelle on explique ce qui suit, pour faire entendre ce qui précède, ou ce qui précède, pour faire entendre ce qui suit: elle ouvre, pour ainsi dire, la porte, dit Quintilien, afin que vous passiez d'une idée à une autre ; ex alio in aliud viam proestat, Inst. VIII.6. C'est l'antécédent pour le conséquent, ou le conséquent pour l'antécédent; et c'est toujours le jeu des idées accessoires dont l'une éveille l'autre " (article "Métalepse ", l'Encyclopédie, 1786); Gérard GenETte, Métalepse: de la figure à la fiction, Paris, Éditions du Seuil, 2004.

70. Sur l'image mentale et les arts de la mémoire, voir Frances YATES, L'art de la mémoire, trad. de l'anglais par Daniel Arasse, Paris, Gallimard, 1975, (1éd., 1966) ; Jean-Philippe ANTOINE, «Ad perpetuam memoriam. Les nouvelles fonctions de l'image peinte en Italie, 1250-1400 », Mélanges de l'École française de Rome, $\mathrm{n}^{\circ} 100-2,1988$, p. 541-615, Lina BOLZONI, La rete delle immagini: predicazione in volgare dalle origini a Bernardino di Siena, Turin, Einaudi, 2002.

71. Exécuté en Toscane, probablement entre 1325 et 1375, conservé au Museo San Matteo de Pise, provenant de l'hôpital Santa Chiara (voir Enzo CARLI, Pittura medievale pisana, Milan, Aldo Martello Editore, 1958, cat. II).

72. Provenant de la Pieve di Marti, à Montopoli in Val d'Arno (Pise), conservé au musée diocésain de San Miniato.

73. E. CARLI, Pittura medeivale pisana, op. cit., fig. 103.

74. Dans le panneau de la Confraternita del Cristo Risorto, (seconde moitié du Xve siècle), Savone, oratoire de la Confraternita del Cristo Risorto. 
75. Mitchell B. MERBACK, « The living image of pity : mimetic violence, peace-making and salvific spectacle in the flagellante processions of the later Middle Ages ", dans Debra H. STRICKLAND (dir.), Images of Medieval Sanctity, Essays in honour of Gary Dickson, Leyde, Boston, Brill, 2007, p. 135-178.

76. Bertrand RoUGÉ, «Le tableau efficace. Réflexions sur la performativité de la peinture (religieuse) », dans Alan DiERKENS, Gil BARTHOLEYNS, Thomas GOLSENNE, La performance des images, Bruxelles, Éditions de l'Université de Bruxelles, 2010 p. 157-168. Voir également dans cet ouvrage Jérôme BASCHET, «Images en acte et agir social », p. 9-14 ainsi que Jean WIRTH, « Performativité de l'image?», p. 125-135.

\section{RÉSUMÉS}

À partir du XIII ${ }^{\mathrm{e}}$ siècle, les confréries de flagellants se multiplient en Occident et particulièrement en Italie. Pour accompagner leurs pratiques dévotionnelles centrées sur la discipline, ces confréries se dotent d'une grande variété d'images au sein de laquelle la Flagellation du Christ tient une place particulière. Cet article interroge la constitution d'un réseau d'images de la Flagellation, de supports et de fonctions variés, essentiel pour l'identité comme pour les pratiques dévotionnelles de ces institutions : bannières processionnelles, enluminures, sceaux, retables, etc. Dans un certain nombre de ces représentations sont par ailleurs figurés des flagellants en train de se livrer à la discipline. Il s'agit, à partir de l'étude du rapport mimétique et spéculaire établi par cette image de flagellants dans la Flagellation, de réfléchir à l'efficace de l'image activée dans le contexte de l'exercice de la dévotion.

Beginning in the $13^{\text {th }}$ century, flagellant confraternities spread throughout Western Europe, especially in Italy. In order to support their devotional practices, based on self-discipline, these brotherhoods acquire a large variety of images wherein Flagellation of Christ figures prominently. This paper examines the constitution of a network of images of Flagellation, in a variety of formats and roles, which is essential for the identity and the devotional practices of theses institutions (processional banners, seals, illuminations, altarpieces). In a number of these images, flagellants are represented whipping themselves. This paper seeks to highlight the agency of the image in the context of devotional practice, through the study of the mimetic and specular relationship between flagellants and Christ wounded by the executioners.

\section{INDEX}

Keywords : Aelred de Rievaulx, confraternities, devotion, flagellants, Flagellation of Christ, imitatio Christi, procession, saint Dominic, repraesentatio

Mots-clés : Aelred de Rievaulx, confréries, dévotion, flagellants, Flagellation du Christ, imitatio Christi, procession, saint Dominique, repraesentatio 


\section{AUTEUR}

\section{PAULINE DUCLOS-GRENET}

Pauline Duclos-Grenet a soutenu une thèse en histoire de l'art médiéval à l'Université de Bourgogne sous la direction de Daniel Russo (Centre Georges Chevrier). Son travail, qui porte sur les images du Procès de Jésus dans l'Italie médiévale, articule des questionnements sur les images de justice, de violence, sur la peinture infamante ou encore sur l'anthropologie des images.

Enseignante dans le second degré, elle est également chargée de cours à l'École du Louvre et à PSL. 